\title{
ON THE SYMMETRIC SQUARE: APPLICATIONS OF A TRACE FORMULA
}

\author{
YUVAL Z. FLICKER
}

\begin{abstract}
In this paper we prove the existence of the symmetric-square lifting of admissible and of automorphic representations from the group $\mathrm{SL}(2)$ to the group PGL(3) . Complete local results are obtained, relating the character of an SL(2)-packet with the twisted character of self-contragredient PGL(3)-modules. Our global results relate packets of cuspidal representations of $\mathrm{SL}(2)$ with a square-integrable component, and self-contragredient automorphic PGL(3)modules with a component coming from a square-integrable one. The sharp results, which concern $\operatorname{SL}(2)$ rather than $\mathrm{GL}(2)$, are afforded by the usage of the trace formula. The surjectivity and injectivity of the correspondence implies that any self-contragredient automorphic PGL(3)-module as above is a lift, and that the space of cuspidal SL(2)-modules with a square-integrable component admits multiplicity one theorem and rigidity ("strong multiplicity one") theorem for packets (and not for individual representations). The techniques of this paper, based on the usage of regular functions to simplify the trace formula, are pursued in the sequel [VI] to extend our results to all cuspidal SL(2)-modules and self-contragredient PGL(3)-modules
\end{abstract}

\section{INTRODUCTION}

Let $H$, or $H_{0}$ be the $F$-group SL(2), $G$ the $F$-group PGL(3), $F$ a global field and $\mathbf{A}$ its ring of adeles. Our aim is to study the lifting (or correspondence, or transfer) of automorphic forms of $H(\mathbf{A})$ to those of $G(\mathbf{A})$, defined by the symmetric-square (or adjoint) three-dimensional representation [II] of the dual (or associate, or $L$-) group $\widehat{H}=\operatorname{PGL}(2, \mathrm{C})$ of $H$ in $\widehat{G}=\mathrm{SL}(3, \mathrm{C})$ (see [B], where $\widehat{G}$ is denoted by ${ }^{L} G$ ), by means of a trace formula.

The interest in the symmetric square lifting originates from Shimura's work [Sh]. Let $f(z)=\sum_{1}^{\infty} c_{n} e^{2 \pi i n z}$ be a holomorphic cusp form of weight $k$ and character $\omega$, signify by $\psi$ a primitive Dirichlet character of $\mathbf{Z}$ with $\psi \omega(-1)=$ 1 , and suppose that

$$
\sum c_{n} n^{-s}=\prod_{p}\left(\left(1-a_{p} p^{-s}\right)\left(1-b_{p} p^{-s}\right)\right)^{-1} .
$$

Received by the editors June 1, 1988 and, in revised form, January 11, 1990.

1980 Mathematics Subject Classification (1985 Revision). Primary 10D40, 10D30, 12A67, $12 \mathrm{~A} 85,14 \mathrm{G} 10,22 \mathrm{E} 55$.

The introduction to the present paper serves as an introduction to the six papers in our Symmetric Square project. They are referred to here as [I], ... ,[VI]; see reference list, [I-VI].

Partially supported by an NSF grant and a Seed grant.

I wish to express my deep gratitude to David Kazhdan for his constructive interest. 
Using Rankin's method Shimura [Sh] proved that the Euler product

$$
\begin{aligned}
& \pi^{-3 s / 2} \Gamma(s / 2) \Gamma((s+1) / 2) \Gamma\left(\frac{1}{2}(s-k+2)\right) \\
& \quad \times \prod_{p}\left[\left(1-\psi(p) a_{p}^{2} p^{-s}\right)\left(1-\psi(p) a_{p} b_{p} p^{-s}\right)\left(1-\psi(p) b_{p}^{2} p^{-s}\right)\right]^{-1}
\end{aligned}
$$

is holomorphic everywhere except possibly at $s=k$ or $k-1$.

Since $f$ generates the space of a cuspidal representation $\pi_{0}$ of $\operatorname{GL}(2, \mathbf{A})$ ( $F=Q$, with a discrete-series component $\pi_{0 \infty}$ at $\left.\infty\right)$, this statement can be put in terms of a lifting of automorphic forms compatible with the above dual group homomorphism, which takes the diagonal complex matrix $\left(a_{p}, b_{p}\right)$ to $\left(a_{p}^{2}, a_{p} b_{p}, b_{p}^{2}\right)$, or rather $\left(a_{p} / b_{p}, 1, b_{p} / a_{p}\right)$.

To reformulate Simura's result Gelbart and Jacquet [GJ] put

$$
L_{2}\left(s, \pi_{0 v}, \chi_{v}\right)=L\left(s, \pi_{0 v} \otimes \chi_{v} \times \check{\pi}_{0 v}\right) / L\left(s, \chi_{v}\right)
$$

and

$$
\varepsilon_{2}\left(s, \pi_{0 v}, \chi_{v} ; \psi_{v}\right)=\varepsilon\left(s, \pi_{0 v} \otimes \chi_{v} \times \check{\pi}_{0 v} ; \psi_{v}\right) / \varepsilon\left(s, \chi_{v} ; \psi_{v}\right)
$$

for any representation $\pi_{0 v}$ of $\operatorname{GL}\left(2, F_{v}\right)$ and character $\chi_{v}$ of the multiplicative group $F_{v}^{\times}$of the completion $F_{v}$ of $F$ at a place $v$. Here $\check{\pi}_{0 v}$ denotes the contragredient of $\pi_{0 v}$, and $\psi_{v}$ is a nontrivial additive character of $F_{v}$. The representation $\pi_{0 v}$ is said in [GJ] to L-lift to a representation $\pi_{v}$ of $G\left(F_{v}\right)$ if $\pi_{v}$ is $\sigma$-invariant (namely, $\pi_{v} \simeq{ }^{\sigma} \pi_{v}$, where ${ }^{\sigma} \pi_{v}(g)=\pi_{v}(\sigma(g)$ ) and $\sigma(g)=$ $\mathscr{J}^{t} g^{-1} \mathscr{J}$,

$$
\mathcal{J}=\left(\begin{array}{lll}
0 & & 1 \\
& -1 & \\
1 & & 0
\end{array}\right),
$$

is an involution of $G)$ and $L\left(s, \pi_{v} \otimes \chi_{v}\right)=L_{2}\left(s, \pi_{0 v}, \chi_{v}\right), \varepsilon\left(s, \pi_{v} \otimes \chi_{v} ; \psi_{v}\right)=$ $\varepsilon_{2}\left(s, \pi_{0 v}, \chi_{v} ; \psi_{v}\right)$ for any $\chi_{v}$.

If $\pi_{0}$ is an automorphic representation of $\mathrm{GL}(2, \mathbf{A})$ and $\chi$ is a character of $\mathbf{A}^{\times} / F^{\times}$, put $L_{2}\left(s, \pi_{0}, \chi\right)=\prod_{v} L_{2}\left(s, \pi_{0 v}, \chi_{v}\right)$. The main theorem of [GJ] is obtained on adelizing the method of [Sh]. It asserts that for any cuspidal representation $\pi_{0}$ of $\operatorname{GL}(2, \mathbf{A})$ not of the form $\pi_{0}(\rho)\left(\rho=\operatorname{Ind}\left(\theta ; W_{F}, W_{E}\right)\right)$, the function $L_{2}\left(s, \pi_{0}, \chi\right)$ is entire for all $\chi$. This refines the statement of [Sh], implies that each component $\pi_{0 v}$ of $\pi_{0} \quad L$-lifts to some $\pi_{v}$, and $\pi=\bigotimes \pi_{v}$ is a cuspidal representation of $G(\mathbf{A})$.

Our approach to the lifting is different; it is motivated by the ideas of Saito and Shintani in the base-change theory. Following Shintani, the local lifting is defined by means of character relations, and following Saito, the global (and local) lifting is studied by means of the (twisted) trace formula. It is shown that the above $\pi_{0}$ (cuspidal, not of the form $\pi_{0}(\rho)$ ), lifts to a cuspidal $\pi$. This implies the holomorphy of $L_{2}\left(s, \pi_{0}, \chi\right)=L(s, \pi \otimes \chi)$ for all $\chi$.

To describe our work, let $L(G)$ be the space of automorphic forms on $G=\operatorname{PGL}(3)$; it consists of all slowly increasing complex-valued functions $\phi$ on $G(F) \backslash G(\mathbf{A})$ with a standard [BJ] right invariance property. The group $G(\mathbf{A})$ acts on $L(G)$ by right translation $(r(g) \phi)(h)=\phi(h g)$. The irreducible constituents $\pi$ are called automorphic $G$-modules, or representations. Each such $\pi$ is a restricted tensor product $\otimes \pi_{v}$ of irreducible admissible representations $\pi_{v}$ of the local groups $G_{v}=G\left(F_{v}\right)$, which are unramified for almost all $v$. 
Each irreducible unramified $G_{v}$-module $\pi_{v}$ is isomorphic to the unique unramified subquotient of a $G_{v}$-module $I\left(\left(\mu_{i v}\right)\right)$ normalizedly induced from an unramified character $\left(a_{i j} ; i \leq j\right) \rightarrow \prod_{i} \mu_{i v}\left(a_{i i}\right)$ of the upper triangular subgroup. Although the character $\left(\mu_{i v}\right)$ is not uniquely determined, we obtain a unique conjugacy class $t\left(\pi_{v}\right)=\operatorname{diag}\left(\mu_{i v}(\boldsymbol{\pi})\right)$ (where $\boldsymbol{\pi}$ denotes a local uniformizer of the maximal ideal in the ring $R_{v}$ of integers in $F_{v}$ ) in the dual group $\widehat{G}=\operatorname{SL}(3, C)$ of $G$. The map $\pi_{v} \rightarrow t\left(\pi_{v}\right)$ is a bijection from the set of equivalence classes of irreducible unramified $G_{v}$-modules to the variety of conjugacy classes in $\widehat{G}$. Similar description holds in the case of $H=\operatorname{SL}(2)$, where the automorphic representations $\pi_{0}=\otimes \pi_{0 v}$ have local components $\pi_{0 v}$ which are parametrized, in the unramified case, by conjugacy classes $t\left(\pi_{0 v}\right)$ in the dual group $\widehat{H}=\operatorname{PGL}(2, \mathbf{C})$ of $H$.

We study lifting of automorphic forms of $H(\mathbf{A})$ to those of $G(\mathbf{A})$, which is compatible with the Symmetric Square representation $\lambda_{0}: \widehat{H} \rightarrow \widehat{G}$ of $\widehat{H}=$ $\operatorname{PGL}(2, \mathbf{C})$ in $\widehat{G}=\operatorname{SL}(3, \mathbf{C})$. This is the irreducible three-dimensional representation of $\widehat{H}$; it can be described also as the adjoint representation of $\widehat{H}$ on its Lie algebra; it maps the diagonal matrix $(a, b)$ to the diagonal matrix $(a / b, 1, b / a)$. We say that the automorphic $H$-module $\pi_{0}=\otimes \pi_{0 v}$ lifts to the automorphic $G$-module $\pi=\otimes \pi_{v}$ if $t\left(\pi_{v}\right)=\lambda_{0}\left(t\left(\pi_{0 v}\right)\right)$ for almost all $v$ (where $\pi_{0 v}$ and $\pi_{v}$ are both unramified). Our first global result asserts that each cuspidal H-module lifts to an automorphic G-module. This result is contained in [GJ]. Here, in [IV], all statements are proven in the context of $H$-modules with a square-integrable component; in [VI] they are extended to deal with general $\pi_{0}$ and $\pi$.

But we can obtain more precise results. It is clear that if $\pi$ is a lift, then it is $\sigma$-invariant. Our second global result is a determination of the image of the lifting. Thus if $\pi$ is a cuspidal G-module which is $\sigma$-invariant then it is a lift of a cuspidal H-module (which is not a representation denoted by $\pi_{0}(\rho)$ and associated by the principle of functoriality (see e.g., [B]), to an induced projective two-dimensional representation $\rho=\operatorname{Ind}\left(\theta ; W_{F}, W_{E}\right)$ of the Weil group $W_{F}$ of $\left.F\right)$. The cuspidal $H$-module $\pi_{0}(\rho)$ lifts to the induced, noncuspidal, $\sigma$-invariant $G$-module $I(\pi(\theta / \bar{\theta}), \chi)$. Here $E$ is a quadratic extension of $F$, and $\theta$ is a character of the idele class group $\mathbf{A}_{E}^{\times} / E^{\times}=C_{E} \simeq W_{E}^{a b}$ of $E$. The character $\theta / \bar{\theta}: z \rightarrow \theta(z / \bar{z})$ of $C_{E}$ determines an automorphic representation $\pi(\theta / \bar{\theta})$ of $\mathrm{GL}(2, \mathbf{A})$, whose central character is the unique nontrivial character $\chi$ of $\mathbf{A}^{\times} / F^{\times}$which is trivial on the norm subgroup $N_{E / F} \mathbf{A}_{E}^{\times}$. The Levi subgroup of the maximal parabolic subgroup of $G$ is isomorphic to $\mathrm{GL}(2)$, hence $\pi(\theta / \bar{\theta})$ determines the induced $G$-module $I(\pi(\theta / \bar{\theta}), \chi)$. Finally the trivial $H$-module lifts to the trivial $G$-module. This gives a complete description of the image. Indeed, any $\sigma$-invariant automorphic $G$-module which is not in the above list, namely it does not have a trivial component, it is not cuspidal and not of the form $I(\pi(\theta / \bar{\theta}), \chi)$, must be of the form $I\left(\pi_{1}, 1\right)$, namely normalizedly induced from a discrete-series $\mathrm{GL}(2, \mathbf{A})$-module $\pi_{1}$ with a trivial central character, and such $I\left(\pi_{1}, 1\right)$ are not obtained by the lifting. Again, here in [IV] all global statements are proven in the context of $G$-modules $\pi$ with a local component $\pi_{v}$ which is a lift (see below) of a square-integrable $H_{0 v}$-module $\pi_{0 v}$; in [VI] these statements are extended to all $\pi$. 
The notion of lifting which we use is in fact a strong one, in terms of all places. Namely we define local lifting of irreducible $H_{v}$-modules to such $G_{v}$ modules, and show that if $\pi_{0}$ lifts to $\pi$, then $\pi_{0 v}$ lifts to $\pi_{v}$ for all places $v$. The definition of local lifting, which is too complicated to recall here, is formulated in terms of identities of characters of representations. It generalizes the notion of lifting of unramified local representations described above. Yet we will mention here that the character relations compare the twisted character of $\pi_{v}$, which is a $\sigma$-stable function, with the character of the packet of $\pi_{0 v}$, which is a stable function. The definitions imply that in the local lifting it is not a single $H_{v}$-module $\pi_{0 v}$ which lifts to $\pi_{v}$, but it is the packet of $\pi_{0 v}$ which lifts. As usual, the packet of an $H_{v}$-module $\pi_{0 v}$ is defined to be the set of admissible irreducible $H_{v}$-modules of the form $\pi_{0 v}^{g}$ ( $g$ in $\left.\mathrm{GL}\left(2, F_{v}\right)\right)$, where $\pi_{0 v}^{g}(h)=\pi_{0 v}\left(g^{-1} h g\right) \quad\left(h\right.$ in $\left.H_{v}\right)$.

Given local packets $P_{v}$ for each place $v$ of $F$ such that $P_{v}$ contains an unramified $H_{v}$-module $\pi_{0 v}^{0}$ for almost all $v$, we define the global packet $P$ to be the set of $H(\mathbf{A})$-modules $\otimes \pi_{0 v}$ with $\pi_{0 v}$ in $P_{v}$ for all $v$ and $\pi_{0 v}$ equivalent to $\pi_{0 v}^{0}$ for almost all $v$. We say that the packet is automorphic, or cuspidal, if it contains such a representation of $H(\mathbf{A})$. In the case of $G$, more generally for $\mathrm{GL}(n)$ and $\mathrm{PGL}(n)$, packets consist of a single term. We are now in a position to state the main lifting theorem. The lifting defines a bijection from the set of packets of cuspidal representations of $H(\mathbf{A})$ to the set of $\sigma$-invariant $G(\mathbf{A})$-modules which are cuspidal or of the form $I(\pi(\theta / \bar{\theta}), \chi)$. This permits the transfer of two well-known theorems for $G=\operatorname{PGL}(3)$, to the context of $H=$ $\mathrm{SL}(2)$. The first is a rigidity theorem for cuspidal representation of $\mathrm{SL}(2)$. It asserts that if $\pi_{0}=\otimes \pi_{0 v}$ and $\pi_{0}^{\prime}=\bigotimes \pi_{0 v}^{\prime}$ are cuspidal $H$-modules and $\pi_{0 v} \simeq$ $\pi_{0 v}^{\prime}$ for almost all $v$, then $\pi_{0}$ and $\pi_{0}^{\prime}$ define the same packet. The analogous statement for $G$ is proven in [JS]. The second application is multiplicity one theorem for $\mathrm{SL}(2)$. It asserts that each cuspidal representation of $\mathrm{SL}(2, \mathbf{A})$ occurs in the cuspidal spectrum of $L(H)$ with multiplicity one. The analogous statement for $\mathrm{GL}(n)$ is well known. Note that the rigidity theorem holds for packets, but not for individual representations. There do exist two inequivalent cuspidal $H$-modules which are equivalent almost everywhere.

Other corollaries to the lifting theorem assert that a $\sigma$-invariant cuspidal $G$-module cannot have a component of the form $I\left(\pi_{1 v}, 1\right)$, where $\pi_{1 v}$ is a square-integrable representation of $\operatorname{GL}\left(2, F_{v}\right)$. Further, if $\pi_{0}$ is a cuspidal $\mathrm{GL}(2)$-module with a local component $I\left(\nu_{v}^{t}, \nu_{v}^{-t}\right), t \geq 0$, normalizedly induced from the character $\left(\begin{array}{ll}a & * \\ 0 & b\end{array}\right) \rightarrow|a / b|_{v}^{t}$ of the upper triangular subgroup, then we conclude as in [GJ] that $t<\frac{1}{4}$. The estimate $t<\frac{1}{2}$ is easy, and the equality $t=0$ is asserted by the Ramanujan conjecture for GL(2). As a final corollary we note that for cuspidal $\pi_{0}$ not of the form $\pi_{0}(\rho)$, since the $L$-function $L_{2}\left(s, \pi_{0}, \chi\right)$ is equal to $L(s, \pi \otimes \chi)$, where $\pi$ is the lift of $\pi_{0}$, we conclude, as noted above, that it is entire for each character $\chi$ of $\mathbf{A}^{\times} / F^{\times}$.

There are six papers [I] , ... , [VI] in our Symmetric Square project, arranged according to their logical interdependence. An attempt has been made to isolate different ideas or techniques and make them as independent as possible. Their contents are as follows. The basic definitions of local lifting of unramified and ramified representations are given in [II]. To study the $\sigma$-invariant $G$-modules $\pi$ not obtained by the lifting we introduce in [II] the the map $\lambda_{1}: \widehat{H}_{1} \rightarrow \widehat{G}$, 
where $\widehat{H}_{1}=\mathrm{SL}(2, \mathrm{C})$ is the dual group of $H_{1}=\operatorname{PGL}(2)=\mathrm{SO}(3)$, in addition to the map $\lambda_{0}: \widehat{H}_{0} \rightarrow \widehat{G}$ mentioned above. We then introduce the dual maps $\tilde{\lambda}_{i}^{*}: \mathbf{H}_{G} \rightarrow \mathbf{H}_{i}$ from the Hecke algebra $\mathbf{H}_{G}$ of spherical functions on $G$ to the Hecke algebras $\mathbf{H}_{i}$ of $H_{i}(i=0,1)$.

These definitions are suggested by the work of [I], where the technical local transfer needed for our study is discussed. There we define a norm map $\gamma=N \delta$ from the set of stable $\sigma$-conjugacy classes of $\delta$ in $G(F)$ to the set of stable conjugacy classes of $\gamma$ in $H(F)$. We transfer smooth compactly supported functions $f_{v}$ on $G\left(F_{v}\right)$ to such $f_{0 v}$ on $H\left(F_{v}\right)$. The definition is based on matching stable orbital integrals. Similar discussion is carried out for the transfer from $G$ to $H_{1}$. Proposition 4.5 of [I] asserts that the unit elements $f_{0 v}^{0}$ of $\mathbf{H}_{0}$ and $f_{v}^{0}$ of $\mathbf{H}$ have matching (stable) orbital integrals. The proof there, due to Langlands, uses buildings. It will be interesting to find a simpler proof for it. The analogous statement of (unstable) transfer of $f_{v}^{0}$ to the unit element $f_{1 v}^{0}$ of $\mathbf{H}_{1}$, namely that they have matching orbital integrals, can be proven by analogous local proof (this has recently been done in [J]), and it is reduced in [V] to the global statement (implicit in [V, Proposition 6], but not yet proven independently) that: any $\sigma$-invariant cuspidal $G$-module $\pi$ has no local component of the form $\pi_{v}=I\left(\pi_{1 v}, 1\right)$, where $\pi_{1 v}$ is a trivial or squareintegrable $H_{1 v}$-module, at any finite place $v$ of odd residual characteristic. The results of [I] are summarized in its introduction.

In [III] we give the global tool for the study of the lifting, an identity of trace formulae. First we compute the trace formula for $G(\mathbf{A})$ twisted by the outer automorphism $\sigma$. Since $\sigma$ does not leave all parabolic subgroups of $G$ invariant, we have to introduce in $\left[F_{1}\right]$ a modification of the truncation used by Arthur to obtain the trace formula. The subsequent computation of the twisted trace formula was carried out in [CL], from which we quote (in [III, §2]), the contribution from the Eisenstein series. Thus in [III] we compute explicitly all terms in the twisted formula, stabilize it, and compare it with a sum of trace formulae for $H=\mathrm{SL}(2)$ and $H_{1}=\operatorname{PGL}(2)$. The formulae in [III] are greatly simplified by the introduction of regular functions (see below).

In [IV, $\S 1]$, we give an approximation argument to deduce from the global identity of trace formulae the local (hence also global) results. It is a new argument. It replaces the technique of [L], which relies on the theory of spherical functions. The new argument is based on the usage of what we call regular functions, which are not spherical but in fact lie in the Hecke algebra with respect to an Iwahori subgroup. Their main property is that they both isolate the representations with a vector fixed by an Iwahori subgroup and their support is easy to control and work with, in contrast to that of spherical function. The approximation (or separation) argument given here applies in any rank one situation (since there are only finitely many reducibility points of principal series representations in this case) and does not use spherical functions at all, except the case where $f_{0 v}$ is the unit element $f_{0 v}^{0}$ of $\mathbf{H}_{0}$ and $f_{v}$ is the unit element in $\mathbf{H}_{G}$, which is recorded in [I, §4]. For further comments see the lines preceding Proposition 1.8.2 below.

In deriving the main theorems in [IV, §2], we use the immediate twisted analogue of Kazhdan's fundamental study of characters [K]. This is formulated in [II, §3]; it is not proven here since the proof is entirely parallel to that of [K] 
and requires no new ideas (cf. $\left[\mathrm{F}_{3}\right]$ in the case of any reductive group). The only nonimmediate result needed to twist $[\mathrm{K}]$ is the analogue of [K, Appendix]. This is done in $\left[\mathrm{F}_{3},(\mathrm{I} .4)\right]$, in general; the special case needed in this paper is done here in [IV, (1.8)].

In [III, §3.7], together with [IV, (1.6.3)], we give a new argument for the comparison of trace formulae for functions $f=\otimes f_{v}$ such that the transer $f_{1 u}$ of $f_{u}$ vanishes for some $u$. This new argument uses the regular functions mentioned above to annihilate the undesirable terms in the trace formula. It replaces the technique of [L], which relies on the computations of singular and weighted orbital integrals and the study of their asymptotic behaviour, and the correction technique of $\left[\mathrm{F}_{0}\right]$. In $[\mathrm{VI}]$ this argument is pursued to give a simple proof of the comparison of trace formulae for all test functions $f$. Thus in [IV] we deal only with automorphic $H$-modules with an elliptic component, but in [VI] we deal with all cuspidal $H$-modules.

The method of [VI] establishes by simple means trace formulae comparisons in other rank one situations. This method may generalize to deal with groups of arbitrary rank and give a simple proof of any trace formulae comparisons for general test functions, but we do not do this here. It affords a simple proof of the base-change lifting for $\mathrm{GL}(2)$ (see $\left[\mathrm{F}_{5}\right]$ ), and its analogues for the quasi-split unitary groups $U(2, E / F)$ and $U(3, E / F)$. See $\left[\mathrm{F}^{\prime}\right]$, where the automorphic and admissible representations of $U(2), U(3)$ are classified, and compared with those of the related general linear groups, and both rigidity and multiplicity one theorems for $U(2), U(3)$, are proven. Perhaps it should be noted here that the approach of $\left[\mathrm{F}^{\prime}\right]$-reducing the study of the representation theory of $U(3, E / F)$ to base-change lifting to $\mathrm{GL}(3, E)$-was found by direct analogy with the techniques of the present paper, whose publication was delayed (for no good reason). Shortly before $\left[F_{1}^{\prime}\right]$ was written, reference [25] in Langlands $\left[\mathrm{L}_{1}\right]$ claimed to have established the endoscopic lifting from $U(2)$ to $U(3)$ by stabilizing the trace formula for $U(3)$ alone. But such a technique is conceptually insufficient for that purpose, as explained in $\left[\mathrm{F}_{3}^{\prime}, \S 4.6\right.$, p. 562].

Our results and some of the techniques are described in $\left[F_{2}\right]$. The present work is an expanded version of the preliminary draft $\left[F_{1}\right]$.

\section{Approximation}

1.1. Lifting. Let $G$ be a reductive group over a number field $F$ with an anisotropic center. Let $L=L(G(F) \backslash G(\mathbf{A}))$ denote the space of slowly increasing functions $\psi$ on $G(F) \backslash G(\mathbf{A})$ with standard conditions of right invariance (see, e.g. [BJ, (4.2)]. The group $G(\mathbf{A})$ acts on $L$ by $(r(g) \psi)(h)=\psi(h g)$. An automorphic representation is an irreducible invariant subquotient, in the sense of [BJ, (4.6)], of $L$. Denote by $L_{0}$ the subspace of all cuspidal functions $\psi$ in $L$. The space $L_{0}$ decomposes as a direct sum of irreducible invariant representations, called cuspidal. Every irreducible representation of $G(\mathbf{A})$ factors as a restricted product $\pi=\otimes \pi_{v}$ over all primes $v$ of local admissible irreducible representations $\pi_{v}$. For almost all places $\pi_{v}$ is unramified.

Put $G=\operatorname{PGL}(3), H=H_{0}=\mathrm{SL}(2), H_{1}=\operatorname{PGL}(2)$. By a discrete-series representation of any of these groups we mean a cuspidal or one-dimensional automorphic representation. The notion of local lifting for unramified representations with respect to the dual-groups homomorphisms $\lambda_{0}: \widehat{H} \rightarrow \widehat{G}, \lambda_{1}: \widehat{H}_{1} \rightarrow \widehat{G}$ 
is defined in [II, (1.6)]. It was generalized to deal with any local representation and formulated in terms of characters in [II, (2.3) and (2.6)]. We write $\pi_{v}=\lambda_{i}\left(\pi_{i v}\right)$ when $\pi_{i v}$ lifts to $\pi_{v}$ with respect to $\lambda_{i}$.

1.1.1. Normalization. Let $\pi=\otimes \pi_{v}$ be a $\sigma$-invariant representation of $G(\mathbf{A})$. Namely $\pi$ is equivalent to the representation ${ }^{\sigma} \pi(g)=\pi(\sigma g)$ of $G(\mathbf{A})$. Then there exists an intertwining operator $A$ on the space of $\pi$ with $A \pi(g) A^{-1}=$ $\pi(\sigma g)$ for all $g$ in $G(\mathbf{A})$. Assume that $\pi$ is irreducible. Then by Schur's lemma the operator $A^{2}$, which interwines $\pi$ with itself, is a scalar which we normalize to be equal to 1 . Fix a nontrivial additive character $\psi$ of $\mathbf{A} \bmod F$. Denote by $\psi$ the character of the upper triangular unipotent subgroup $N(\mathbf{A})$, defined by $\psi(n)=\psi(x+z)$, where

$$
n=\left(\begin{array}{lll}
1 & x & y \\
0 & 1 & z \\
0 & 0 & 1
\end{array}\right) .
$$

Note that $\psi(\sigma n)=\psi(n)$. Assume that $\pi$ is nondegenerate, namely it can be realized in a space of (Whittaker) functions $W$ on $G(\mathbf{A})$ which satisfy $W(n g k)=\psi(n) W(g)$ for all $g$ in $G(\mathbf{A}), n$ in $N(\mathbf{A})$, and $k$ in a compact open subgroup of $G\left(\mathbf{A}_{f}\right)$, depending on $W$; here $\mathbf{A}_{f}$ denotes the ring of finite adeles; $\pi$ acts by $\pi(g) W(h)=W(h g)$. Then ${ }^{\sigma} \pi$ is nondegenerate and can be realized in the space of functions ${ }^{\sigma} W(g)=W(\sigma g), W$ in the space of $\pi$. We take $A$ to be the operator on the space of $\pi$ which maps $W$ to ${ }^{\sigma} W$.

This gives a normalization of the intertwining operator $A$, which is also local in the following sense. Each component $\pi_{v}$ of $\pi$ is nondegenerate and can be realized in a space of Whittaker functions $W_{v}$ (with $W_{v}\left(n_{v} g_{v} k_{v}\right)=$ $\psi_{v}\left(n_{v}\right) W_{v}\left(g_{v}\right)$, where $\psi_{v}$ is the restriction of $\psi$ to $\left.N_{v}=N\left(F_{v}\right)\right)$. Moreover, each $W$ is a finite linear combination of products $\otimes W_{v}$; where for almost all $v$ the component $W_{v}$ is the (unique up to a scalar multiple) unramified (i.e., right $K_{v}=G\left(R_{v}\right)$-invariant) Whittaker function. Now we can write $A$ as a product $\otimes A_{v}$ over all places, where $A_{v}$ is the operator intertwining $\pi_{v}$ with ${ }^{\sigma} \pi_{v}$, which maps $W_{v}$ to ${ }^{\sigma} W_{v}$. This is the normalization of the local operators used below. We put $\pi_{v}(\sigma)=A_{v}$, and $\pi_{v}\left(f_{v} \times \sigma\right)$ for the operator $\pi_{v}\left(f_{v}\right) A_{v}$, when $\pi_{v}$ is a nondegenerate representation.

In the special case when $\pi_{v}$ is unramified, there exists a unique Whittaker function $W_{v}$ in the space of $\pi_{v}$ with respect to $\psi_{v}$ (provided $\psi_{v}$ is unramified), with $W_{v}\left(k_{v}\right)=1$ for $k_{v}$ in $K_{v}=G\left(R_{v}\right)$. It is mapped by $\pi_{v}(\sigma)=A_{v}$ to ${ }^{\sigma} W_{v}$, which satisfies ${ }^{\sigma} W_{v}\left(k_{v}\right)=1$ for all $k_{v}$ in $K_{v}$ since $K_{v}$ is $\sigma$-invariant. Namely $A_{v}$ maps the unique $K_{v}$-fixed vector $W_{v}$ in the space of $\pi_{v}$ to the unique $K_{v}$-fixed vector ${ }^{\sigma} W_{v}$ in the space of ${ }^{\sigma} \pi_{v}$, and we have ${ }^{\sigma} W_{v}=W_{v}$. Hence $A_{v}$ acts as the identity on the $K_{v}$-fixed vectors, and our local normalization is the one used in the study of spherical functions in [II, $\S 1.2]$. If

We take $\pi(\sigma)$ to be the identity if $\pi$ is the trivial representation of $G(\mathbf{A})$.

$$
\pi=I(1 ; P, G)=\left\{\phi: G(\mathbf{A}) \rightarrow \mathbf{C} ; \phi(p g)=\delta_{p}^{1 / 2}(p) \phi(g), g \in G(\mathbf{A}), p \in P(\mathbf{A})\right\}
$$

is the $G(\mathbf{A})$-module normalizedly induced from the trivial representation of the maximal parabolic subgroup $P$ of $G$ of type $(2,1) \quad\left(\delta_{P}\right.$ is the modular function of $P$ ), then the conjugate representation ${ }^{\sigma} \pi$ is the induced $I\left(1 ;{ }^{\sigma} P, G\right)$ 
from the trivial representation of the parabolic ${ }^{\sigma} P$ of type $(1,2)$. In this case we define $\pi(\sigma)$ by $(\pi(\sigma) \phi)(g)=\phi(\sigma g)$.

1.2. Definition. The automorphic representation $\pi_{i}=\bigotimes \pi_{i v}$ of $H_{i}(\mathbf{A})$ (quasi-) lifts to the automorphic representation $\pi=\otimes \pi_{v}$ of $G(\mathbf{A})$ if $\pi_{v}=\lambda_{i}\left(\pi_{i v}\right)$ for (almost) all $v$.

1.2.1. Example. Let $\pi_{1}=\bigotimes \pi_{1 v}$ be an automorphic representation of $H_{1}(\mathbf{A})$. Let $\pi=\otimes \pi_{v}$ be the representation $I\left(\pi_{1}\right)$ of $G(\mathbf{A})$ normalizedly induced from the representation $\pi_{1} \otimes 1$ of its maximal parabolic subgroup $P=M N$. Note that the Levi factor $M$ of $P$ is isomorphic to $\operatorname{GL}(2, \mathbf{A})$ and $\pi_{1}$ defines a representation of $M$ which is trivial on the center. Then $\pi$ is irreducible, and also $\sigma$-invariant, since (1) ${ }^{\sigma} \pi$ is the representation $I\left(\check{\pi}_{1}\right)$ induced from the contragredient $\check{\pi}_{1}$ of $\pi_{1}$, (2) $\check{\pi}_{1}$ is equivalent to $\pi_{1}$, being a representation of $H_{1}=\operatorname{PGL}(2)$. We have that $\pi_{1}$ quasi-lifts to $\pi$ by virtue of [II, (1.6)].

1.2.2. Example. Let $F$ be a local or global field. Let $K$ be a quadratic extension of $F$. Put $C_{K}$ for the Weil group $W_{K / K}$ (it is isomorphic to $K^{\times}$if $K$ is local, and to $\mathbf{A}_{K}^{\times} / K^{\times}$if $K$ is global). Put $C_{K}^{1}$ for the kernel of the norm map from $C_{K}$ to $C_{F}$. Similarly we have $K^{1}$ and $\mathbf{A}_{K}^{1}$. Note that $\mathbf{A}_{K}^{1} / K^{1} \simeq C_{K}^{1}$. The Weil group $W_{K / F}$ is an extension of $\mathrm{Gal}(K / F)$ by $C_{K}$. It can be described as the group generated by the $z$ in $C_{K}$ and $\tau$ with $\tau^{2}$ in $C_{F}-N_{K / F} C_{K}$, under the relation $\tau z=\bar{z} \tau$; the bar indicates the action of the nontrivial element of $\operatorname{Gal}(K / F)$.

Let $\theta$ be a character of $C_{K}$. The projective two-dimensional induced representation $I(\theta)=\operatorname{Ind}\left(\theta ; W_{K / K}, W_{K / F}\right)$ of $W_{K / F}$ in the dual group $\widehat{H}=$ $\operatorname{PGL}(2, \mathrm{C})$ of $H=\mathrm{SL}(2)$ can be realized as

$$
W_{K / K} \ni z \rightarrow\left(\begin{array}{cc}
\theta(z) & 0 \\
0 & \theta(\bar{z})
\end{array}\right) \times z, \quad \tau \rightarrow\left(\begin{array}{cc}
0 & 1 \\
-1 & 0
\end{array}\right) \times \tau .
$$

It depends only on the restriction of $\theta$ of $C_{K}^{1}$. Denote by $\chi$ the nontrivial (quadratic) character of $C_{F}$ whose kernel is $N_{K / F} C_{K}$.

If $F$ is local and $\theta=\bar{\theta}(\bar{\theta}$ is the character defined by $\bar{\theta}(z)=\theta(\bar{z})$ for all $z$ ), then there is a character $\mu$ of $C_{F}$ with $\theta(z)=\mu(N z)(N z=z \bar{z})$. We define the representation $\pi(\theta)$ of $\operatorname{GL}(2, F)$ associated with $\theta$, or $I(\theta)$, to be the induced representation $I(\mu, \mu \chi)$. Also we define the packet $\left\{\pi_{0}\right\}=\left\{\pi_{0}(\theta)\right\}$ of representations of $H(F)$ associated with $I(\theta)$ to be the set of irreducible subquotients of the representations (normalizedly) induced from the character $\left(\begin{array}{cc}a & b \\ 0 & a^{-1}\end{array}\right) \rightarrow \chi(a)$ of the Borel subgroup. It consists of two elements. In this case $\left\{\pi_{0}(\theta)\right\}$ is independent of $\theta$ since $\theta$ is trivial on $C_{K}^{1}$.

If $F$ is global, for almost all places $v$ of $F$ the character $\theta$ is unramified, and then at an inert $v$ we have $\theta_{v}=1$ on $K_{v}^{1}$. At $v$ which splits in $K / F$ the restriction of $I(\theta)$ to $W_{K_{V} / F_{V}}$ is a direct sum of two characters, and this defines a representation induced from the Borel subgroup, again denoted by $\pi\left(\theta_{v}\right)$ or $\left\{\pi_{0}\left(\theta_{v}\right)\right\}$ (a packet in the case of $H\left(F_{v}\right)$ ). It is well known (see, e.g., [LL]) that there exists an automorphic representation $\pi_{0}(\theta)$ of $\operatorname{SL}(2, \mathbf{A})$ whose components for (almost) all $v$ are in the above $\left\{\pi_{0}\left(\theta_{v}\right)\right\}$. 
Applying the map $\lambda_{0}=\operatorname{Sym}^{2}$ to $I$, we get the representation

$$
z \rightarrow(\theta(z / \bar{z}), 1, \theta(\bar{z} / z)) \times z, \quad \tau \rightarrow\left(\begin{array}{lll}
0 & & 1 \\
& -1 & \\
1 & & 0
\end{array}\right) \times \tau,
$$

of $W_{K / F}$ in $\widehat{G}=\operatorname{SL}(3, \mathrm{C})$. It is the direct sum of the two-dimensional representation $\operatorname{Ind}\left(\theta(z / \bar{z}) ; W_{K / K}, W_{K / F}\right)$ and the one-dimensional representation $x \rightarrow \chi(x)$ of $W_{F / F}$, where $\chi$ is the quadratic character of $W_{F / F}$ associated with the quadratic extension $K / F$ by class field theory.

This direct sum parametrizes the representation $\pi$ of $G(\mathbf{A})$ induced from the representation $\pi^{*} \times \chi$ of a maximal parabolic $P$. Here $\pi^{*}=\pi^{*}\left(\theta^{\prime}\right)$ is a $\mathrm{GL}(2)$-module, where we put $\theta^{\prime}(z)=\theta(z / \bar{z})$. The representation $\pi$ is $\sigma$ invariant, since ${ }^{\sigma} \pi$ is the representation induced from $\check{\pi}^{*} \times \chi^{-1}$. But $\chi$ is of order two, and for our $\pi^{*}$ of the form $\pi^{*}\left(\theta^{\prime}\right)$, the contragredient $\check{\pi}^{*}$ is $\pi^{*} \otimes \chi \simeq \pi^{*}$ [LL]. It follows from [II, (1.6)] that $\pi_{0}$ quasi-lifts to $\pi$.

More generally, if $\pi_{0}$ is an automorphic representation parametrized by a map $\rho: W_{F} \rightarrow \widehat{H}$, and $\pi$ is one parametrized by the composition $\lambda_{0} \circ \rho$ of $\rho$ and $\lambda_{0}: \widehat{H} \rightarrow \widehat{G}$, then it is clear that $\pi_{0}$ quasi-lifts to $\pi$ upon restricting $\rho$ to the local Weil group $W_{F_{v}}$. But it is not clear that given $\pi_{0}$, there exists such $\pi$ which is the quasi-lift of $\pi_{0}$. For this we need to use the trace formula, which yields also local lifting at all places and global lifting.

1.3. Terms. To formulate the identity of traces of $\sigma$-invariant representations in $L(G(F) \backslash G(\mathbf{A}))$, in $L(H(F) \backslash H(\mathbf{A}))$ and $L\left(H_{1}(F) \backslash H_{1}(\mathbf{A})\right)$, with which we study the lifting, we now describe the terms which appear in it.

$$
I=\sum_{\pi} \prod_{v} \operatorname{tr} \pi_{v}\left(f_{v} \times \sigma\right) .
$$

This sum is taken over all equivalence classes of discrete-series (see (1.1)) representations $\pi=\bigotimes \pi_{v}$ of $G$. The multiplicity one theorem for $\operatorname{GL}(3)$ is used here to write this sum without multiplicities, as each equivalence class of $\pi$ consists of a single representation.

$$
I^{\prime}=\sum_{K} \sum_{\theta} \prod_{v} \operatorname{tr} I_{v}\left(\left(\pi_{v}(\theta / \bar{\theta}), \chi_{v}\right) ; f_{v} \times \sigma\right) .
$$

Here the first sum is over all quadratic extensions $K$ of $F$, and $\chi$ denotes the quadratic character of $F^{\times} \backslash A^{\times}$whose kernel is $N_{K / F}\left(A_{K}^{\times}\right)$. The second sum is over all characters $z \rightarrow \theta(z / \bar{z})$ of $W_{K / K}$ which are not of order 2, up to the equivalence $\theta \simeq \theta^{-1}$.

$$
I^{\prime \prime}=\sum_{\eta} \prod_{v} \operatorname{tr} I_{v}\left(\eta, f_{v} \times \sigma\right) .
$$

The sum is over unordered triples $\eta=\{\chi, \mu \chi, \mu\}$, where $\chi, \mu$ are characters of $W_{F / F}$ of order 2 (not 1 ), and $\chi \neq \mu$. The induced representation $I(\eta)$ from the character $\eta$ of the Borel subgroup can also be described in the form $I(\pi(\theta / \bar{\theta}), \chi)$, where $z \rightarrow \theta(z / \bar{z})$ is as in $I^{\prime}$ but of order 2 , and then there exists a character $\mu$ of $W_{F / F}$ of order 2 with $\theta(z / \bar{z})=\mu(z \bar{z})$.

$$
I_{1}=\sum_{\pi_{1}} \prod_{v} \operatorname{tr} \pi_{1}\left(f_{1 v}\right)
$$


and

$$
I_{1}^{\prime}=\frac{1}{2} \sum_{\pi_{1}} \prod_{v} \operatorname{tr} I_{v}\left(\left(\pi_{1 v}, 1\right) ; f_{v} \times \sigma\right) .
$$

Both sums extend over the equivalence classes (consisting of a single element each) of the discrete-series representations of $H_{1}=\operatorname{PGL}(2)$.

$$
I_{0}=\sum_{\left\{\pi_{0}\right\}} m\left(\left\{\pi_{0}\right\}\right) \prod_{v} \operatorname{tr}\left\{\pi_{0 v}\right\}\left(f_{0 v}\right) .
$$

The sum ranges over almost all packets of discrete-series representations of $H=$ $\operatorname{SL}(2)$. A local packet $\left\{\pi_{0 v}\right\}$ was defined in [II, (1.2)]. A global packet $\left\{\pi_{0}\right\}$ is defined if we are given a local packet $P_{v}$ for all $v$, which contains an unramified element $\pi_{0 v}^{0}$ for almost all $v$. It consists of all representations $\otimes \pi_{0 v}$ with $\pi_{0 v}$ in $P_{v}$ for all $v$ and $\pi_{0 v}=\pi_{0 v}^{0}$ for almost all $v$. We say that $\left\{\pi_{0}\right\}$ is a discreteseries packet if it contains an automorphic representation which appears in the discrete-series, and a cuspidal packet if it contains a cuspidal representation. The sum of $I_{0}$ ranges over all discrete-series packets $\left\{\pi_{0}\right\}$ not of the form $\pi(\theta)$. If $\left\{\pi_{0}\right\}$ consists of the one-dimensional representation, put $m\left(\left\{\pi_{0}\right\}\right)=1$. It is shown in [LL, (6.4)], that each element in a cuspidal packet $\left\{\pi_{0}\right\}$ not of the form $\pi(\theta)$, is cuspidal. Moreover, if $\pi_{0}^{\prime}$ and $\pi_{0}^{\prime \prime}$ are two elements in such $\left\{\pi_{0}\right\}$, then their multiplicities $m\left(\pi_{0}^{\prime}\right)$ and $m\left(\pi_{0}^{\prime \prime}\right)$ in the cuspidal spectrum $L_{0}(H(F) \backslash H(\mathbf{A}))$ are equal and denoted by $m\left(\left\{\pi_{0}\right\}\right)$.

$$
I_{0}^{\prime}=\sum_{K} \sum_{\theta} \prod_{v} \operatorname{tr}\left\{\pi_{0}\left(\theta_{v}\right)\right\}\left(f_{0 v}\right) .
$$

$K$ ranges over the quadratic extensions of $F$. The second sum ranges over the characters $z \rightarrow \theta(z / \bar{z})$ of $W_{K / K}$ whose order is not (1 or 2), up to the equivalence $\theta \simeq \theta^{-1}$. Implicit in $I_{0}^{\prime}$ and $I_{0}^{\prime \prime}$ is a result of [LL, (6.6), and (6.3) with $G^{\prime}=H, A=A_{F}=1$ ] which asserts that each member of $\left\{\pi_{0}(\theta)\right\}$ appears in the discrete spectrum with multiplicity at most one.

$$
I_{0}^{\prime \prime}=\sum_{\{\mu, \chi, \mu \chi\}} \prod_{v} \operatorname{tr}\left\{\pi_{0}\left(\theta_{v}\right)\right\}\left(f_{0 v}\right) .
$$

The sum ranges over all packets $\left\{\pi_{0}\right\}$ of $H(\mathbf{A})$ of the form $\left\{\pi_{0}(\theta)\right\}$, where $\theta$ is a character of $K^{\times} \backslash \mathbf{A}_{K}^{\times}$with $(\theta / \bar{\theta})^{2}=1$ but $\theta / \bar{\theta} \neq 1$, where $K$ is a quadratic extension of $F$. Note that $K$ defines a character $\chi$ of $\mathbf{A}^{\times} / F^{\times}$ of order 2 , and since $\theta / \bar{\theta}=\bar{\theta} / \theta$ there exists a character $\mu$ of $\mathbf{A}^{\times} / F^{\times}$of order 2 (in particular unitary) with $\theta(z / \bar{z})=\mu(z \bar{z})$, and $\mu \neq \chi$. The packet $\pi_{0}(\theta)$ is uniquely determined by the unordered triple $\{\mu, \chi, \mu \chi\}$ of quadratic characters.

1.1.1. Fix a representation $\pi_{v}$ of $G\left(F_{v}\right)$ for almost all $v$. The rigidity theorem for $\mathrm{GL}(3, \mathbf{A})$ of [JS] implies that each of $I, I_{1}^{\prime}, I^{\prime}$ and $I^{\prime \prime}$ consists of at most one entry $\pi$ with the above components for almost all $v$, and, moreover, at most one of the four terms has such an entry.

1.4. Lemma. Let $F$ be a local field. Suppose $\pi=I\left(\pi^{\prime}, \mu\right)$ is a $\sigma$-invariant representation of $G(F)$ induced from a maximal parabolic subgroup, where $\pi^{\prime}$ is a square-integrable representation of the $2 \times 2$ factor and $\mu$ is a unitary character. Then either $\mu=1$ and $\pi^{\prime}$ is a representation $\pi_{1}$ of $H_{1}(F)$, or 
$\mu$ is a character of order 2 and $\pi^{\prime}$ is a representation of the form $\pi(\theta / \bar{\theta})$ of $\mathrm{GL}(2, F)$, where $\theta$ is a character of the quadratic extension $K$ of $F$ determined by $\mu$.

Remark. The lemma and its proof are valid also in the case where $F$ is global and $\pi$ is an automorphic representation of $G(\mathbf{A})$.

Proof. By definition of induction ${ }^{\sigma} \pi$ is $I\left(\check{\pi}^{\prime}, \mu^{-1}\right)$, where $\check{\pi}^{\prime}$ is the contragredient of $\pi^{\prime}$. Since $I\left(\pi^{\prime}, \mu\right)$ is tempered, the square-integrable data $\left(\pi^{\prime}, \mu\right)$ is uniquely determined. Hence, as $I\left(\pi^{\prime}, \mu\right)$ is equivalent to $I\left(\check{\pi}^{\prime}, \mu^{-1}\right)$, our $\pi^{\prime}$ is equivalent to $\check{\pi}^{\prime}$ and $\mu=\mu^{-1}$. The central character of $\pi^{\prime}$ is $\mu=\mu^{-1}$ since $\pi$ is a representation of $\operatorname{PGL}(3)$. If $\mu=1$ then $\pi^{\prime}$ is a representation of $\mathrm{GL}(2, F)$ with a trivial central character. If $\mu \neq 1$, since $\check{\pi}^{\prime} \simeq \pi^{\prime} \otimes \mu$ we have $\pi^{\prime} \simeq \pi^{\prime} \otimes \mu$. This implies (by [LL]) that $\pi^{\prime}$ is parametrized by an induced representation $\operatorname{Ind}\left(\theta^{\prime} ; W_{K}, W_{F}\right)$ of the Weil group $W_{F}$. The central character of $\pi^{\prime}\left(\theta^{\prime}\right)$ is $\left.\mu \cdot \theta^{\prime}\right|_{F^{\times}}$, where $\left.\theta^{\prime}\right|_{F^{\times}}$denotes the restriction of the character $\theta^{\prime}$ of $K^{\times}$to $F^{\times}$. On the other hand, this central character is equal to $\mu$. Hence $\theta^{\prime}=1$ on $F^{\times}$, and there exists a character $\theta$ on $K^{\times}$with $\theta^{\prime}(z)=\theta(z / \bar{z}) \quad(z$ in $K^{\times}$), as required.

1.5. Regularity. Let $F$ be a nonarchimedean local field, $n$ a positive integer, $\mu$ a unitary character of $A_{0}(R)=\left\{\left(a, a^{-1}\right) ;|a|=1\right\}$; we denote by $(a, b)$ the diagonal matrix $\left(\begin{array}{ll}a & 0 \\ 0 & b\end{array}\right)$. We write $H, G$ for $H(F), G(F)$, etc. Recall that we write $\Phi(\gamma, f)$ or $\Phi_{f}(\gamma)$ for the orbital integral of $f$ at $\gamma$, and $F_{f}(\gamma)$ or $F(\gamma, f)$ for $\Delta(\gamma) \Phi(\gamma, f)$. Let $\pi$ be a local uniformizer of $F$.

Definition. Let $S$ be the open closed set of $\gamma$ in $H$ which are conjugate to $\left(a \pi^{n}, a^{-1} \pi^{-n}\right)$ in $H$, where $a$ lies in $R^{\times}$. The function $f_{0}$ is called regular of type $(n, \mu)$ if $f_{0}$ is supported on $S$ and $F\left(\left(a \pi^{n}, a^{-1} \pi^{-n}\right), f_{0}\right)=\mu(a)^{-1}$ for every $a$ in $R^{\times}$. When $\mu=1$ we say that $f_{0}$ is regular of type $n$.

Analogous definition applies to $f_{1}$ and $f$. For example, we say that $f$ is regular of type $(n, \mu)$ if the value of $f$ at $\delta$ in $G$ is zero unless $\delta$ is $\sigma$ conjugate to $\left(a \pi^{n}, 1,1\right)$, and then $F\left(\left(a \pi^{n}, 1,1\right), f\right)=\mu(a)^{-1}$.

1.5.1. Modules of coinvariants [BZ]. Let $(\pi, V)$ be an admissible $G$-module, $N$ the upper triangular subgroup, $V_{N}$ the quotient of $V$ by the span of $n \cdot v-v$ ( $n$ in $N, v$ in $V$ ). It is an $A$-module, as $A$ normalizes $N$. The associated representation of $A$ is denoted by ' $\pi_{N}$, and we put $\pi_{N}=\delta^{-1 / 2}{ }^{\prime} \pi_{N}$, where $\delta(a, b, c)=|a / c|^{2}$. Since $\pi$ is $\sigma$-invariant and $N$ is $\sigma$-invariant, $V_{N}$ is an $A \times\langle\sigma\rangle$-module, and $\pi_{N}$ is a $\sigma$-invariant representation of $A$. It is an admissible representation, studied in [BZ]. The function $\delta$ is introduced to preserve unitarity [BZ, p. 444, last line]. Its character on $A \times \sigma$ is denoted by $\chi\left(\pi_{N}\right)$, so that

$$
\operatorname{tr} \pi_{N}(f \times \sigma)=\int_{A} f(a)\left(\chi\left(\pi_{N}\right)\right)(a) d a,
$$

for any smooth compactly supported function $f$ on $A$. If $\pi_{i}$ are all of the irreducible subquotients of $\pi_{N}$ (repeated with multiplicities) which are equivalent to their $\sigma$-conjugates, then $\operatorname{tr} \pi_{N}=\sum_{i} \operatorname{tr} \pi_{i}$. The Deligne-Casselman theorem [C] easily generalizes to our case, and asserts that $\chi_{\pi}(\delta)=\chi\left({ }^{\prime} \pi_{N}\right)(\delta)$ (these are the unnormalized characters), hence $\left(\Delta \chi_{\pi}\right)(\delta)=\left(\chi\left(\pi_{N}\right)\right)(\delta)$, for $\delta=(a b, 1, b)$ with $|a|<1$. 
Similar definitions hold for representations $\pi_{0}$ of $H$. Again $N$ is the upper triangular subgroup (of $H$ ), ' $\pi_{0 N}$ is defined as above and so is $\pi_{0 N}$, where $\delta\left(a, a^{-1}\right)=|a|^{2}$. The Theorem of [C], which is stated for the unnormalized characters, implies that $\left(\Delta \chi_{\pi_{0}}\right)(\gamma)=\left(\chi\left(\pi_{0 N}\right)\right)(\gamma)$ at $\gamma=\left(a, a^{-1}\right)$ with $|a|<1$. For any function $f_{0}$ on $H$ put

$$
f_{0 N}(\gamma)=\delta^{1 / 2}(\gamma) \int_{H(R)} \int_{N} f_{0}\left(k^{-1} \gamma n k\right) d n d k .
$$

1.5.2. Computation. Let $f_{0}$ be a regular function of type $(n, \mu)$, and $\pi_{0}$ an irreducible representation of $H$. Then, using the Weyl integration formula (see [II]), we have

$$
\begin{aligned}
\operatorname{tr} \pi_{0}\left(f_{0}\right) & =\operatorname{tr} \pi_{0 N}\left(f_{0 N}\right)=\frac{1}{2} \int_{A_{0}} \chi\left(\pi_{0 N}\right)(a) F\left(a, f_{0}\right) d a \\
& =\int_{A_{0}(R)} \chi\left(\pi_{0 N}\right)\left(\left(a \pi^{n}, a^{-1} \pi^{-n}\right)\right) \mu^{-1}(a) d a .
\end{aligned}
$$

If $\mu$ is ramified, that is, $\mu \neq 1$, then $\operatorname{tr} \pi_{0}\left(f_{0}\right)$ vanishes unless $\pi_{0}$ is a subquotient of the induced representation $I_{0}\left(\mu^{\prime}\right)$ of $H$, in the notations of [II, (2.8)], where $\mu^{\prime}$ is a character of $A_{0} \simeq F^{\times}$with $\mu^{\prime}=\mu$ on $A_{0}(R) \simeq R^{\times}$. Then

$$
\left(\chi\left(\pi_{0 N}\right)\right)\left(a, a^{-1}\right)=\mu^{\prime}(a)+\mu^{\prime}\left(a^{-1}\right),
$$

and $\operatorname{tr} \pi_{0}\left(f_{0}\right)$ is equal to $\left(\mu^{\prime}\right)\left(\pi^{n}\right)$ unless $\mu^{2}=1$ on $A_{0}(R)$. If $\mu^{2}=1$ but $\mu^{\prime 2} \neq 1$ then $I_{0}\left(\mu^{\prime}\right)$ is irreducible and $\operatorname{tr} \pi_{0}\left(f_{0}\right)$ is equal to $z^{n}+z^{-n}$, where $z=\mu^{\prime}\left(\pi^{n}\right)$. If $\mu^{\prime 2}=1$ but $\mu^{\prime} \neq 1$ then $I_{0}\left(\mu^{\prime}\right)$ is reducible and $\operatorname{tr} \pi_{0}\left(f_{0}\right)=$ $\mu^{\prime}\left(\pi^{n}\right)$, where $\pi_{0}$ is any of the two irreducible quotients of $I_{0}\left(\mu^{\prime}\right)$.

Suppose that $\mu=1$. In this case, if $\operatorname{tr} \pi_{0}\left(f_{0}\right) \neq 0$ then $\pi_{0}$ is a constituent of $I_{0}\left(\mu^{\prime}\right)$ where $\mu^{\prime}$ is unramified. Hence $\pi_{0}$ has a nonzero vector fixed under the action of an Iwahori subgroup, by [B', Lemma 4.7]. We have $\operatorname{tr}\left(I_{0}\left(\mu^{\prime}\right)\right)\left(f_{0}\right)=$ $\mu^{\prime}\left(\boldsymbol{\pi}^{n}\right)+\mu^{\prime}\left(\boldsymbol{\pi}^{n}\right)^{-1}$, and this is the value of $\operatorname{tr} \pi_{0}\left(f_{0}\right)$ when $I_{0}\left(\mu^{\prime}\right)$ is irreducible. Reducibility occurs when $z=\mu^{\prime}(\boldsymbol{\pi})$ is equal to $q=|\boldsymbol{\pi}|^{-1}, q^{-1}$ or -1 . If $z=q$ or $q^{-1}$, then the composition series of $I_{0}\left(\mu^{\prime}\right)$ consists of the trivial representation 1 and the special representation $\mathrm{sp}$. Then $\operatorname{tr} 1\left(f_{0}\right)=q^{n}$ and $\operatorname{tr} \operatorname{sp}\left(f_{0}\right)=q^{-n}$. If $z=-1$ then $I_{0}\left(\mu^{\prime}\right)$ is the direct sum of two irreducibles $\pi_{0}$, and $\operatorname{tr} \pi_{0}\left(f_{0}\right)=(-1)^{n}$ for each of them.

1.5.3. Twisted computation. Let $f$ be a regular function of type $(n, \mu)$, and $\pi$ a $\sigma$-invariant irreducible representation of $G$. The twisted Weyl integration formula (see [II]) implies that

$$
\operatorname{tr} \rho(f \times \sigma)=\int_{R^{\times}}\left(\chi\left(\pi_{N}\right)\right)\left(\left(a \pi^{n}, 1,1\right) \times \sigma\right) \mu^{-1}(a) d a,
$$

and this does not vanish only when $\pi$ is a subquotient of a representation $I\left(\mu^{\prime}\right)$ of $G$ induced from a character $\mu^{\prime}=\left(\mu_{1}, \mu_{2}, \mu_{2}\right)$ of $A$, such that there is a character ' $\mu$ of $F^{\times}$with ' $\mu=\mu$ on $R^{\times}$and $\mu^{\prime}(a, b, c)=\mu_{1}(a) \mu_{2}(b) \mu_{3}(c)$ is equal to ' $\mu(a / c)$. Namely $\mu^{\prime}=\left({ }^{\prime} \mu, 1,{ }^{\prime} \mu^{-1}\right)$. As in [II], $\chi\left(\pi_{N}\right)((a, b, c) \times \sigma)$ is the sum of ${ }^{\prime} \mu(a / c)$ and ${ }^{\prime} \mu(c / a)$. Put $z={ }^{\prime} \mu\left(\pi^{n}\right)$. Then $\operatorname{tr} I\left(\mu^{\prime}\right)(f \times \sigma)$ is equal to $z^{n}$, unless $\mu^{2}=1$ when it is equal to $z^{n}+z^{-n}$. These are the values of $\operatorname{tr} \pi(f \times \sigma)$ if $\pi$ lies in irreducible $I\left(\mu^{\prime}\right)$. But the list of (1.4) and the reduciblility results of [BZ] imply that if $I\left(\mu^{\prime}\right)$ is $(\sigma$-invariant and) reducible, 
then $I\left(\mu^{\prime}\right)$ is $I\left(\nu^{-1}, 1, \nu\right)$ or $I\left(\chi \nu^{-1 / 2}, 1, \chi \nu^{1 / 2}\right)$, where $\chi$ is a character of $F^{\times}$with $\chi^{2}=1$, and $\nu$ denotes the character $\nu(x)=|x|$. Then $\mu=1$ or $\mu=\chi$ (respectively), and $\operatorname{tr} I\left(\mu^{\prime}\right)(f \times \sigma)=z^{n}+z^{-n}$, and $z$ equals $q$ or $q^{1 / 2} \chi(\pi) ; \chi(\pi)$ equals 1 or -1 . In the first case, where $z=q$, the composition series of $I\left(\mu^{\prime}\right)$ consists of (1) the trivial representation 1 , and $\operatorname{tr} 1(f \times \sigma)=$ $q^{n}$; (2) the Steinberg representation st, and $\operatorname{trst}(f \times \sigma)=q^{-n}$; and some other non- $\sigma$-invariant irreducibles. In the second case, where $z=\chi(\pi) q^{1 / 2}$, the composition series of $I\left(\mu^{\prime}\right)$ consists of two $\sigma$-invariant irreducibles. Let $\operatorname{sp}(\chi)$ and $1(\chi)$ denote the special and one-dimensional subquotients of the induced representation $I\left(\nu^{1 / 2}, \nu^{-1 / 2}\right) \otimes \chi$ of $\mathrm{GL}(2)$. Let $P$ denote a maximal proper parabolic subgroup of $G$; its Levi factor is isomorphic to $\operatorname{GL}(2)$. Then the composition series of $I\left(\mu^{\prime}\right)$ consists of the irreducibles $I_{P}(\operatorname{sp}(\chi), 1)$ and $I_{P}(1(\chi), 1)$ induced from $P$, and

$$
\operatorname{tr}\left(I_{P}(\operatorname{sp}(\chi), 1)\right)(f \times \sigma)=z^{-n}, \quad \operatorname{tr}\left(I_{P}(1(\chi), 1)\right)(f \times \sigma)=z^{n} .
$$

It is clear that when $\mu=1$ and $\operatorname{tr} \pi(f \times \sigma) \neq 0$, then the irreducible $\pi$ has a vector fixed by the action of an Iwahori subgroup, again by [B', Lemma 4.7].

1.6. Comparison. Let $F$ be a global field. Suppose that $f=\otimes f_{v}$ and $f_{i}=$ $\otimes f_{i v}$ are products of smooth compactly supported functions $f_{v}$ and $f_{i v}$ on $G\left(F_{v}\right)$ and $H_{i}\left(F_{v}\right)$. Suppose $f_{v}$ and $f_{i v}$ are the unit elements $f_{v}^{0}$ and $f_{i v}^{0}$ in the Hecke algebras $\mathbf{H}$ and $\mathbf{H}_{i}$ [II, (1.2)] of $G\left(F_{v}\right)$ and $H_{i}\left(F_{v}\right)$ for almost all $v$. Suppose that $f_{i v}=\lambda_{i}^{*}\left(f_{v}\right)$ for all $v$ in the notations of [I, $\left.\S 3\right]$, namely $\Phi_{f_{v}}^{\text {st }}(\delta)=\Phi_{f_{0 v}}^{\text {st }}(\gamma)$ whenever $\gamma=N \delta$, and a similar statement of matching orbital integrals for $f_{1 v}$. It is shown in [I, §3] that for each $f_{v}$ there exists $f_{i v}$ and for each $f_{i v}$ there exists $f_{v}$ with $f_{i v}=\lambda_{i}^{*}\left(f_{v}\right)$, and in [I, §4] that $f_{0 v}^{0}=\lambda_{0}^{*}\left(f_{v}^{0}\right)$. Since it has not been shown as yet that $f_{1 v}^{0}=\lambda_{1}^{*}\left(f_{v}^{0}\right)$ (this is done in [J]) we make the following restriction on $f$. Denote by $\Phi_{f_{v}}^{l a b}$ the unstable, orbital integral of $f_{v}$ defined in [I, (3.5)].

1.6.1. Restriction. There is a place $u^{\prime}$ of $F$ such that $\Phi_{f_{v}}^{l a b}(\delta)=0$ for all $\sigma$-regular $\delta$ in $G\left(F_{v}\right)$ at $v=u^{\prime}$; note that $u^{\prime}$ can be archimedean or nonarchimedean.

Recall that $\delta$ is called $\sigma$-regular (resp. $\sigma$-elliptic, $\sigma$-split) if $\gamma=N \delta$ is regular (resp. elliptic, split) in $H\left(F_{v}\right)$, where $N$ is the norm map defined in [I].

Under this restriction we can choose $f_{1 u}$, to be 0 , hence $f_{1}=\otimes f_{1 v}$ to be 0 , and $I_{1}=0$. Consequently, we do not need to know that $f_{1 v}^{0}=\lambda_{1}^{*}\left(f_{v}^{0}\right)$ for almost all $v$.

Fix a finite place $u\left(\neq u^{\prime}\right)$ of $F$. Fix $f_{v}, f_{0 v}$ for all $v \neq u$ to be matching as in (1.6) such that (1.6.1) is satisfied at $u^{\prime}$. Put $f^{u}=\otimes f_{v}, f_{0}^{u}=\bigotimes f_{0 v}$ (product over $v \neq u$ ). Proposition 3.5 of [III] asserts

1.6.2. Lemma. There exists an absolutely integrable function $d(z)$ on the unit circle in $\mathbf{C}^{\times}$, and a positive integer $n^{\prime}$ depending on $f^{u}, f_{0}^{u}$, such that if $f_{u}$, $f_{0 u}$ are regular of type $n, n \geq n^{\prime}$, then

$$
I+\frac{1}{2} I^{\prime}+\frac{1}{4} I^{\prime \prime}+\frac{1}{2} I_{1}^{\prime}-I_{0}-\frac{1}{2} I_{0}^{\prime}-\frac{1}{4} I_{0}^{\prime \prime}-\frac{1}{2} I_{1}=J,
$$

where

$$
J=\int d(z)\left(z^{n}+z^{-n}\right) d^{\times} z, \quad|z|=1 .
$$


Indeed, $\operatorname{tr} I_{0}\left(\mu, f_{u}\right)=z^{n}+z^{-n}$, where $z=\mu(\pi)$.

Remark. As the one-dimensional representation which appears in $I_{0}$ lifts to the one-dimensional representation in $I$, we may assume that $I$ and $I_{0}$ consist of cuspidal representations only.

\subsubsection{Proposition. $J$ is equal to 0 .}

Proof. The sum of the I's on the left of $J$ can be written as

$\sum_{i} c_{i}\left(z_{i}^{n}+z_{i}^{-n}\right)+a_{0} q^{n}+a_{1} q^{-n}+a_{2} q^{n / 2}+a_{3} q^{-n / 2}+a_{4}\left(-q^{1 / 2}\right)^{n}+a_{5}\left(-q^{-1 / 2}\right)^{n}$,

where $a_{i}$ and $c_{i}$ are complex numbers, the sum is absolutely convergent, and $c_{i}$ is a sum of $\operatorname{tr} \pi^{u}\left(f^{u} \times \sigma\right), \operatorname{tr} \pi_{0}^{u}\left(f_{0}^{u}\right)$ etc. with coefficient $1, \frac{1}{2}$ or $\frac{1}{4}$, over the $\pi^{u}, \ldots$ such that $\pi=\pi^{u} \otimes \pi_{u}, \ldots$ appears in the sum of $I, \ldots$, where $\pi_{u}=I\left(\mu^{\prime}\right)$ determines $z_{i}$ as in (1.5.2), (1.5.3) (with $\left.\mu=1\right)$. Here $z_{i} \neq$ $q, q^{-1}, q^{1 / 2}, q^{-1 / 2},-q^{1 / 2},-q^{-1 / 2}$. We shall use the following comments. All representations in the trace formula have unitary components. Hence each $z_{i}$ lies in the compact subset $X^{\prime}=X^{\prime}(q)$ in $\mathbf{C}$ which is the union of the unit circle $|z|=1$ and the real segments $q^{-1} \leq z \leq q$ and $q^{-1} \leq-z \leq q$. Let $X=X(q)$ be the quotient of $X^{\prime}$ by the equivalence relation $z^{-1} \sim z$. Then $X$ is a compact Hausdorff space. Let $B=B(q)$ be the space spanned over C by the functions $f_{n}(z)=z^{n}+z^{-n}$ on $X$, where $n \geq 0$. It is closed under multiplication, contains the scalars, and separates points of $X$. Moreover, if $f$ lies in $B$ then its complex conjugate $\bar{f}$ does too. Hence the Stone-Weierstrass theorem implies the following

Lemma. $B$ is dense in the sup norm in the space of complex-valued continuous functions on $X$.

Put $F_{n}(z)=q^{2} f_{n+3}(z)-q f_{n+2}(z)-q f_{n+1}(z)+f_{n}(z)$. Since the sum over $i$ and the integral $J$ are absolutely convergent, (1.6.2) implies that for every $n>n^{\prime}$ we have

$$
\sum_{i} c_{i} F_{n}\left(z_{i}\right)+a_{0} F_{n}(q)+a_{2} F_{n}\left(q^{1 / 2}\right)+a_{4} F_{n}\left(-q^{1 / 2}\right)=\int d(z) F_{n}(z) d^{\times} z .
$$

Had we replaced $q$ by $q^{-1}$ in the definition of $F_{n}(z)$, the $a_{0}, a_{2}, a_{4}$ here would be replaced by $a_{1}, a_{3}, a_{5}$. We write the left side here in the form $\sum_{i \geq 0} b_{i} F_{n}\left(z_{i}\right)$, where now the $z_{i}$ are distinct elements in $X$. The sum ranges over all $i \geq 0$ such that $b_{i} \neq 0$. Our aim is to show that the sum is empty. Suppose that it is not empty.

We first show that $\left|z_{i}\right|=1$ for all $i$. If this is false, we may assume that $z_{0}>1$. Let $n^{\prime \prime}>n^{\prime}$ be an odd integer. For every $n \geq 0$ we obtain from (1.6.2) the identity

$$
\sum_{i \geq 0} b_{i} F_{n}^{\prime \prime}\left(z_{i}\right) f_{n}\left(z_{i}^{3 n^{\prime \prime}}\right)=\int d(z) F_{n}^{\prime \prime}(z) f_{n}\left(z^{3 n^{\prime \prime}}\right) d z .
$$

Writing $t_{j}$ for the distinct $z_{i}^{3 n "}$, and $t$ for $z^{3 n^{\prime \prime}}$, we obtain (for every $n \geq 0$ )

$$
\sum_{j \geq 0} b_{j}^{\prime} f_{n}\left(t_{j}\right)=\int_{|t|=1} d^{\prime}(t) f_{n}(t) d t .
$$


The sum ranges over distinct points of $X\left(q^{3 n^{\prime \prime}}\right)$. We have $b_{0}^{\prime}=b_{0} F_{n^{\prime \prime}}\left(z_{0}\right) \neq 0$ since $F_{n^{\prime \prime}}\left(z_{0}\right) \neq 0$ and $z_{0}^{3 n^{\prime \prime}} \neq z_{i}^{3 n^{\prime \prime}}$ for all $z_{i}$. We may assume that $b_{0}^{\prime}=1$. The absolute convergence of the sum and integral implies that there is $c>0$ with $\int_{|t|=1}\left|d^{\prime}(t)\right| d t \leq c$, and for each $\varepsilon>0$ there is $m>0$ so that $\sum_{j>m}\left|b_{j}^{\prime}\right|<$ $\varepsilon$. The Lemma implies that there is $f$ in $B\left(q^{3 n^{\prime \prime}}\right)$ with $f\left(t_{0}\right)=1$ which is bounded by 2 on $X\left(q^{3 n^{\prime \prime}}\right)$, whose value at $t_{1}, \ldots, t_{m}$ and on $|t|=1$ is very small. Evaluating the linear function $(*)$ at $f$, which is a finite linear combination of $f_{n}$ 's we conclude that $b_{0}=b_{0}^{\prime}=0$.

We know that $\left|z_{i}\right|=1$ for all $i$. Let $S$ be the quotient of the unit circle $S^{\prime}$ by the relation $z \sim z^{-1}$. In particular, $a_{0}, \ldots, a_{5}$ are zero, and we can write the identity of $(1.6 .2)$ in the form

$$
\sum_{i \geq 0} b_{i} f_{n}\left(z_{i}\right)=\int d(z) f_{n}(z) d^{\times} z
$$

$z$ and $z_{i}$ are in $S$. Arguing as above we have $c>0$ with $\int_{|z|=1}|d(z)| d z \leq c$, and $m>0$ with $\sum_{i \geq m}\left|b_{i}\right|<\varepsilon$. Moreover there is $f$ in $B$ with $f\left(z_{0}\right)=1$ which is bounded by 2 on $S$, whose value outside of a small neighborhood of $z_{0}$ is small. The only problem is that $(* *)$ holds only when $n \geq n^{\prime}$. But this is easy to overcome. Take $k$ larger than the sum of $n^{\prime}$ and the degree of $f$, such that $z_{0}^{k}$ is close to one. Then $\left|z^{k}+z^{-k}\right| \leq 2$ on $S$, and we can apply (**) with $f_{n}$ replaced by $g(z)=f(z)\left(z^{k}+z^{-k}\right)$ to obtain a contradiction to $b_{0} \neq 0$. This establishes the proposition.

1.7. Proposition. Let $V$ be a finite set of places of $F$ including the archimedean places. Fix a conjugacy class $t_{v}$ in $\widehat{H}$ for all $v$ outside $V$. For any choice of matching $f_{v}$ and $f_{0 v}\left(=\lambda_{0}^{*}\left(f_{v}\right)\right)$ for $v$ in $V$ which satisfy restriction (1.6.1) we have

$$
I+\frac{1}{2} I^{\prime}+\frac{1}{4} I^{\prime \prime}+\frac{1}{2} I_{1}^{\prime}=I_{0}+\frac{1}{2} I_{0}^{\prime}+\frac{1}{4} I_{0}^{\prime \prime}+\frac{1}{2} I_{1},
$$

where $I, I_{0}, \ldots$ are defined by products over $v$ in $V$ only, the sums in $I, I_{0}, \ldots$ are taken only over $\pi,\left\{\pi_{0}\right\}, \ldots$ whose component at $v$ outside $V$ is unramified and parametrized by the conjugacy class $\lambda_{0}\left(t_{v}\right)$ in $\widehat{G}$ or $t_{v}$ in $\widehat{H}$.

Proof. The proof of (1.6.2) applied inductively to the elements in a set $U$ of places outside $V$, implies that $\sum_{i} c_{i} \prod f_{0 v}^{\vee}\left(t_{i v}\right)=0$, where the product ranges over $v$ outside $V \cup U$, the sum is over all sequences $\left\{t_{i v} ; v\right.$ outside $\left.V\right\}$ in $\widehat{H}$ with $t_{i v}=t_{v}$ for $v$ in $U$, and $c_{i}$ is defined by the difference of the left and right sides of (1.7.1) (corresponding to the sequence $\left.\left\{t_{i v}\right\}\right)$. We have to show that $c_{i}=0$ for all $i$. Suppose $c_{0} \neq 0$. Choose a positive $m$ with $\sum\left|c_{i}\right|<\frac{1}{2}\left|c_{0}\right|$ (sum over $i \geq m$ ), and a set $U$ disjoint from $V$ so that for each $1 \leq i<m$ there is $u$ in $U$ with $t_{i u} \neq t_{u}$. Applying $(*)$ with this $U$ and with $f_{0 v}^{v}=1$ (thus $f_{0 v}=f_{0 v}^{0}$ ) for all $v$ outside $V \cup U$, we obtain a contradiction which proves the proposition.

1.7.2. Theorem. Under the conditions of (1.7) at most one of the sums $I, I^{\prime}$, $I^{\prime \prime}, I_{1}^{\prime}$ is nonempty, and consists of a single summand.

Proof. This follows from the rigidity theorem of [JS].

1.7.3. Corollary. Fix a nonarchimedean $u$ in $V$ and an irreducible $H_{u}$-module $\pi_{0 u}^{\prime}$ which is not supercuspidal. Then (1.7.1) holds where the products in $I, I_{0}, \ldots$ 
range only over $v \neq u$ in $V$, and the sums in $I, I_{0}, \ldots$ are the subsums of those specified in (1.7) where $\left\{\pi_{0}\right\}$ has the above component $\left\{\pi_{0 u}^{\prime}\right\}$ at $u$, and $\pi$ has component $\pi_{u}$ which lies in the induced representation of $G_{u}$ which is the lift of the induced representation $I_{0}\left(\mu^{\prime}\right)$ of $H_{u}$ containing $\pi_{0 u}^{\prime}$.

Proof. Denote by $\mu$ the restriction of $\mu^{\prime}$ to ' $R_{u}^{\times}$. The case of $\mu=1$ is dealt with in Proposition 1.6.2 (or (1.7)). That of $\mu^{2}=1$ is the same. If $\mu^{2} \neq 1$ let $f_{0 u}^{\prime}$ be a regular function of type $(n, \mu)$, and consider $f_{0 u}=f_{0 u}^{\prime}+\bar{f}_{0 u}^{\prime}$; note that $\bar{f}_{0 u}^{\prime}$ is of type $\left(n, \mu^{-1}\right)$. Then $\operatorname{tr} \pi_{0 u}\left(f_{0 u}\right)$ vanishes unless $\pi_{0 u}$ is irreducible and induced $I_{0}\left({ }^{\prime} \mu\right)$ from a character ' $\mu$ of $F_{u}^{\times}=A_{0 u}$ whose restriction to $R_{u}^{\times}$is $\mu$, then $\operatorname{tr} \pi_{0 u}\left(f_{0 u}\right)$ equals $z^{n}+z^{-n}$ for a suitable $z$. As the same observations apply on the twisted side, applying the Stone-Weierstrass theorem as in (1.6.2) the corollary follows.

It is convenient to rewrite (1.7.1) in the form

$$
\sum_{\pi} n(\pi) \prod_{v} \operatorname{tr} \pi_{v}\left(f_{v} \times \sigma\right)=\sum_{\left\{\pi_{0}\right\}} n\left(\left\{\pi_{0}\right\}\right) \prod_{v} \operatorname{tr}\left\{\pi_{0 v}\right\}\left(f_{0 v}\right) .
$$

On the left the sum ranges over a set of $\sigma$-invariant automorphic representations of $G(\mathbf{A})$, not necessarily in the discrete spectrum, specified in (1.3). The sum on the right is over automorphic packets $\left\{\pi_{0}\right\}$ of $H(\mathbf{A})$. The coefficients are either $\frac{1}{2}, \frac{1}{4}$ or integers. The products are taken over $v$ in $V$, as specified in (1.7) and (1.7.3).

1.7.5. Corollary. Suppose $w$ in $V$ is such that each $\pi_{w}$ in (1.7.4) is a lift of some $\left\{\pi_{0 w}\right\}$. Fix a packet $\left\{\pi_{0 w}^{\prime}\right\}$. Then (1.7.4) (or (1.7.1)) remains valid if $w$ is omitted from the products over $v$, and the sums are taken over $\left\{\pi_{0}\right\}$ with component $\left\{\pi_{0 w}^{\prime}\right\}$ at $w$ and $\pi$ with components $\lambda_{0}\left(\left\{\pi_{0 w}^{\prime}\right\}\right)$ at $w$.

Proof. Our assumption implies that (1.7.1) can be expressed in the form

$$
\sum_{\left\{\pi_{0 w}\right\}} c\left(\left\{\pi_{0 w}\right\}\right) \operatorname{tr}\left\{\pi_{0 w}\right\}\left(f_{0 w}\right)=0,
$$

since it is shown in [I, §3], that for each $f_{0 w}$ there is a matching $f_{w}$ (with $f_{0 w}=$ $\left.\lambda_{0}^{*}\left(f_{w}\right)\right)$. If $\left\{\pi_{0 w}\right\}$ contains a square-integrable representation then we take $f_{0 w}$ to be a pseudo-coefficient and conclude that the corresponding coefficient is zero. Hence we may assume that each $\left\{\pi_{0 w}\right\}$ in the sum is the set of (one or two) constituents of an induced representation $I_{0}\left(\mu_{w}\right)$. Since the sum is absolutely convergent, the argument of (1.6.3) implies that the coefficients are all zero, as required.

1.7.6. Lemma. (1) The conclusion of (1.7.5) holds at a complex place $w$ of $F$.

(2) If $F$ is totally imaginary then (1.7.1) holds where all archimedean places are omitted (in the sense of (1.7.5)) from $V$; then the sums in (1.7.4) are finite for a fixed choice of $f_{0 v}, f_{v}(v$ in $V, v \neq \infty)$.

Proof. (1) Let $\pi$ be an irreducible admissible $\sigma$-invariant representation of $G(\mathbf{C})$ which appears as a component at a complex place of an automorphic representation on the left of (1.7.4). Since the trivial representation of $H(\mathrm{~A})$ lifts to the trivial representation of $G(\mathbf{A})$, we may assume that either (i) $\pi$ is nondegenerate, in which case it is induced from a character of a Borel subgroup, hence it is the lift of an induced $\pi_{0}$; here we use the description [Vo, Theorem 6.2(f)], 
of nondegenerate (= large) representations of $G(\mathbf{C})$; or (ii) $\pi$ is induced from the trivial representation of a maximal proper parabolic subgroup. Note that $\mathbf{C}^{\times}$has no characters of order two. Indeed, any automorphic representation of $G(\mathbf{A})$ which appears in $I, I^{\prime}$ or $I^{\prime \prime}$ and is not one-dimensional, is either nondegenerate (and each of its local components is nondegenerate), or it is induced from a one-dimensional representation of a maximal parabolic subgroup. In case (ii) $\pi$ is a constituent of a representation $\pi^{\prime}$ of $G(\mathbf{C})$ induced from a Borel subgroup. Well-known results concerning $\mathrm{GL}(2, \mathbf{C})$-modules assert that $\pi^{\prime}$ has a composition series of length two which consists of the irreducible $\pi$ and a $G(\mathbf{C})$-module $\pi^{\prime \prime}$ which is also induced from a Borel subgroup. $\pi^{\prime}$ is the lift of $\pi_{0}^{\prime}$, and $\pi^{\prime \prime}$ of $\pi_{0}^{\prime \prime}$, where $\pi_{0}^{\prime}, \pi_{0}^{\prime \prime}$ are induced representations of $H(\mathbf{C})$. Then $\operatorname{tr} \pi(f \times \sigma)=\operatorname{tr} \pi_{0}^{\prime}\left(f_{0}\right)-\operatorname{tr} \pi_{0}^{\prime \prime}\left(f_{0}\right)$ for all matching $f_{0}, f$, and (1.7.5) applies. Namely in case (ii) we have not verified the assumption of (1.7.5) (which is false since $\pi$ is neither $\lambda_{0}\left(\left\{\pi_{0}^{\prime}\right\}\right)$ nor $\lambda_{0}\left(\left\{\pi_{0}^{\prime}\right\}\right)$, but showed that the proof of (1.7.5) applies nevertheless. Note that for each $f_{0}$ on $H(\mathbf{C})$ there is a matching $f$ on $G(\mathbf{C})$, e.g. by [I, $\S 3]$.

For (2), the sums are finite by a theorem [BJ, 4.3(i), p. 195] which asserts that there are only finitely many automorphic representations $\pi$ of $G(\mathbf{A})$ with a fixed infinitesimal character and a $C$-fixed vector; $C$ is an open compact subgroup of $G\left(\mathbf{A}_{f}\right)$, and $\mathbf{A}_{f}$ denotes the finite adeles. The conditions of this theorem are satisfied in our case since we fixed the archimedean components of the $\pi$ and the $\pi_{0}$, and we choose $f_{v} \quad(v \neq \infty)$ to be invariant under such fixed $C$.

1.8. Density. For a global function $f$ whose components at $u^{\prime}, u^{\prime \prime}$ are supported on the $\sigma$-elliptic regular set, the twisted trace formula takes the form (see [III, (3.2.5)]).

$$
I+\frac{1}{2} I^{\prime}+\frac{1}{4} I^{\prime \prime}+\frac{1}{2} I_{1}^{\prime}=\sum_{\{\delta\}} c_{\gamma} \Phi_{f}(\delta) .
$$

The sum is over all conjugacy classes of elements $\delta$ in $G(F)$ whose norm $\gamma=N \delta$ in $H(F)$ is elliptic regular. The $c_{\gamma}$ are volume factors, see [III, (1.2.1)]. The sum is finite. With analogous conditions on $f_{0}$, the stable trace formula for $H$ takes the form

$$
I_{0}+\frac{1}{2} I_{0}^{\prime}+\frac{1}{4} I_{0}^{\prime \prime}=\sum_{\{\gamma\}} c_{\gamma} \Phi_{f_{0}}^{\text {st }}(\gamma) .
$$

The sum is over all stable conjugacy classes of elliptic regular elements in $H(F)$. The $c_{y}$ are as above and the sum is again finite. The following is a twisted analogue of Kazhdan [K, Appendix].

Proposition. Suppose that $\operatorname{tr} \pi_{u}\left(f_{u} \times \sigma\right)=0$ for all admissible $\pi_{u}$ (which are the component at $u$ of the $\pi$ which make a contribution (1.8.1)). Then the twisted orbital integral $\Phi_{f_{u}}(\delta)$ of $f_{u}$ is 0 for all $\delta$ in $G\left(F_{u}\right)$.

Proof. By virtue of [I, §3] it suffices to consider only $\sigma$-regular $\delta$. Since $G(F)$ is dense in $G\left(F_{u}\right)$ and $\Phi_{f_{u}}(\delta)$ is smooth, it suffices to show that in each neighborhood of $\delta$ in $G\left(F_{u}\right)$ there exists a $\sigma$-regular $\delta_{0}$ in $G(F)$ with $\Phi_{f_{u}}\left(\delta_{0}\right)=0$. We choose such $\delta_{0}$ which is $\sigma$-elliptic at the places $u^{\prime}, u^{\prime \prime}$. We choose $f$ whose components at $u^{\prime}, u^{\prime \prime}$ are supported on the $\sigma$-regular elliptic set, so that (1.8.1) holds, such that the component of $f$ at $u$ is our $f_{u}$, and $\Phi_{f_{v}}\left(\delta_{0}\right) \neq 0$ for all 
$v \neq u$. The assumption of the lemma implies that $\sum_{\{\delta\}} c_{\gamma} \Phi_{f}(\delta)=0$. The sum ranges over all $\sigma$-conjugacy classes of $\sigma$-elliptic regular $\delta$ in $G(F)$. Since $f$ is compactly supported it is clear that the eigenvalues of $N \delta$ lie in a finite set (depending on $f$ ). These eigenvalues determine the stable $\sigma$-conjugacy class of $\delta$. By [I, Corollary 1.5], we may restrict the support of $f^{u}=\bigotimes_{v \neq u} f_{v}$ to have $\Phi_{f u}(\delta)=0$ for all $\delta$ in the sum unless $\delta$ is $\sigma$-conjugate to $\delta_{0}$. Since $\Phi_{f^{u}}\left(\delta_{0}\right) \neq 0$ and $\Phi_{f}\left(\delta_{0}\right)=0$, and $c_{\gamma} \neq 0$, it follows that $\Phi_{f_{u}}\left(\delta_{0}\right)=0$, as required.

We shall now adapt the above techniques to show that corresponding spherical functions have matching stable orbital integrals. However we do not use this result in our work. Our method is new. It is based on the usage of regular functions. \{Perhaps it should be noted that the present paper was written in 1985 , but its publication was delayed. Its method was extended in [FK and $F_{4}$ ] to deal with groups of general rank. As noted in $\left[F_{6}\right.$, p. 3$]$, there is a gap in $\left[F_{4}\right]$. I wish to thank J.-P. Labesse for filling in this gap (by Proposition 8, p. 525, in Duke Math. J. 61 (1990); he also reports that the method was known to him decades ago ... $)$. We checked-but did not write up-that this result can also be proven by a method of Clozel, which is global too (as in [K, Appendix]), but relies instead on properties of spherical, not Iwahori, functions. In fact Clozel writes in [Cl, p. 151, line 3], that his method is the one used in this work. But his assertion is wrong, and in more than one sense. It is said that Langlands proved the matching statement (unpublished; we have not seen this manuscript) by combinatorics on buildings. In any case our method is the simplest.

As in [I, (3.1)], and [II], we write $\lambda_{0}^{*}(f)=f_{0}$ if $f$ and $f_{0}$ are matching (have matching stable orbital integrals), and $\tilde{\lambda}_{0}(f)=f_{0}$ if $f$ and $f_{0}$ are corresponding spherical functions (see [II, $\S 1$ ]; they satisfy $\operatorname{tr} \pi(f \times \sigma)=\operatorname{tr} \pi_{0}\left(f_{0}\right)$ for all unramified $\pi_{0}$ and $\pi$ with $\left.\pi=\lambda_{0}\left(\pi_{0}\right)\right)$.

\subsubsection{Proposition. For each $f$ in $\mathbf{H}$ we have $\lambda_{0}^{*}(f)=f_{0}$ if $\tilde{\lambda}_{0}^{*}(f)=f_{0}$.}

Proof. As in (1.8) it suffices to consider a $\sigma$-regular $\delta_{0}$ in $G(F)$ which is $\sigma$ elliptic at $u^{\prime}, u^{\prime \prime}$, choose $f^{u}=\bigotimes f_{v}(v \neq u)$ whose components at $u^{\prime}, u^{\prime \prime}$ satisfy (1.6.1) and are supported on the $\sigma$-regular set, with $\Phi^{\text {st }}\left(\delta_{0}, f^{u}\right) \neq 0$. The component at $u$ is taken to be a regular function of any type $n$. The function $f_{0}=f_{0}^{u} \otimes f_{0 u}$ is taken in a parallel fashion, so that $f, f_{0}$ have matching orbital integrals. Hence

$$
\sum c_{\gamma} \Phi^{\mathrm{st}}\left(\delta, f_{0}\right)=\sum c_{\gamma} \Phi^{\mathrm{st}}(\delta, f),
$$

where the sums over stable conjugacy classes are finite. Recall from [I, $\S 1]$, that the norm map is a bijection from the set of stable $\sigma$-conjugacy classes in $G(F)$, to the set of stable conjugacy classes in $H(F)$. By (1.8.1) we obtain (1.7.1), which we write as in the proof of (1.6.2) in the form

$$
\sum c\left(\pi_{0 u}\right) \operatorname{tr} \pi_{0 u}\left(f_{0 u}\right)=0 \quad \text { or } \quad \sum c_{i}\left(z_{i}^{n}+z_{i}^{-n}\right)=0 .
$$

As in (1.6.2) we conclude that the coefficients $c_{i}$, or $c\left(\pi_{0 u}\right)$, are zero. In particular we can take the subsum in (1.8.4) over spherical $\pi_{0 u}$ only, and it is equal to zero also when $f_{0 u}, f_{u}$ are replaced by corresponding spherical functions as in our proposition. Hence we obtain (1.8.3) where $f_{0 u}, f_{u}$ are now corresponding spherical functions. As the sums are finite we can reduce the support of the 
component $f_{0 u^{\prime}}$, so that the only entry to the sums in (1.8.3) is $\delta_{0}$. Indeed, a stable $\sigma$-conjugacy class $\delta$ is determined by the eigenvalues of $\delta \sigma(\delta)$. Since $\Phi^{\mathrm{st}}\left(\delta_{0}, f^{u}\right)$ is nonzero by construction, we have $\Phi^{\mathrm{st}}\left(\delta_{0}, f_{u}\right)=\Phi^{\mathrm{st}}\left(N \delta_{0}, f_{0 u}\right)$ for all $\sigma$-regular $\delta_{0}$ (in $G(F)$, hence in $G\left(F_{u}\right)$ ), as asserted.

1.9. Suppose that $F$ is local, $G$ is a reductive group over $F, \pi$ is an admissible representation of $G, C$ is a compact open subsgroup of $F^{\times}, f$ is the quotient of the characteristic function of $C$ by the volume $|C|$ of $C$ with respect to the measure which appears in the definition of $\operatorname{tr} \pi(f)$.

Lemma. $\operatorname{tr} \pi(f)$ is equal to the dimension of the space of $C$-fixed vectors in $\pi$, namely it is a nonnegative integer.

Proof. $\pi(f)$ is the projection on the space of $C$-fixed vectors in $\pi$.

1.9.1. Definition. Let $J$ be a reductive group over a local field, $\pi$ a squareintegrable $J$-module, and $f$ a smooth compactly supported (modulo center) function on $J$. Then $f$ is called a pseudo-coefficient of $\pi$ if $\operatorname{tr} \pi(f)=1$ and $\operatorname{tr} \pi^{\prime}(f)=0$ for any irreducible tempered $J$-module $\pi^{\prime}$ inequivalent to $\pi$.

The existence of pseudo-coefficients for $H=\operatorname{SL}(2)$ is well known. Their existence for any $p$-adic group is proven in Kazhdan [ $\mathrm{K}$, Theorem $\mathrm{K}$ ].

It is illuminating to record here some results of [LL] concerning the sum $I_{0}$, or the discrete spectrum of $L(H(F) \backslash H(\mathbf{A}))$.

1.9.2. Lemma. Every automorphic (cuspidal) representation $\pi_{0}$ of $H(\mathbf{A})$ is contained in an automorphic (cuspidal) representation $\tilde{\pi}$ of $\mathrm{GL}(2, \mathbf{A})$. If $\tilde{\pi}$ and $\tilde{\pi}^{\prime}$ contain $\pi_{0}$ then $\tilde{\pi}^{\prime}=\omega \otimes \tilde{\pi}$ for a character $\omega$ of $\mathbf{A}^{\times}$. If $\tilde{\pi}$ contains $\pi$ and $\pi^{\prime}$ then $\pi^{\prime}=\pi^{h}$ for some $h$ in $\mathrm{GL}(2, \mathbf{A})$, where $\pi^{h}(g)=\pi\left(h^{-1} g h\right)$.

Proof. The first assertion follows from [LL, Lemma 6.2], and the other two are properties of induction, verified in [LL, Lemmas 2.4, 2.5]. Let us recall an argument for the first claim. Extend $\pi_{0}$ to an automorphic representation of $H^{\prime}(\mathbf{A}), H^{\prime}=Z H$, by extending the central character of $\pi_{0}$ to $Z(F) \backslash Z(\mathbf{A})$; $Z$ denotes here the center of $\operatorname{GL}(2)$. Put $\tilde{\pi}=\operatorname{Ind}\left(V_{\pi_{0}} ; H^{\prime}(\mathbf{A}), \widetilde{H}(\mathbf{A})\right), \widetilde{H}=$ $\mathrm{GL}(2)$. Here the space $V_{\pi_{0}}$ of $\pi_{0}$ is a space of automorphic forms. Define a linear functional $l: V_{\pi_{0}} \rightarrow \mathbf{C}$ by $l(\psi)=\psi(1)$. Note that $l(\pi(\gamma) \pi(g) \psi)=$ $\psi(\gamma g)=\psi(g)=l(\pi(g) \psi)$ for all $\gamma$ in $H^{\prime}(F)$ since $\psi$ is automorphic. It suffices to construct an embedding of the space $V_{\tilde{\pi}}$ of $\tilde{\pi}$ into $L(\widetilde{H}(F) \backslash \tilde{H}(\mathbf{A}))$. The induced representation $\tilde{\pi}$ operates by right translation in the space $V_{\tilde{\pi}}$ of functions $f: \widetilde{H}(\mathbf{A}) \rightarrow V_{\pi_{0}}$ which are compactly supported modulo $H^{\prime}(\mathbf{A})$ and satisfy $f(s g)=\pi_{0}(s) f(g) \quad\left(s\right.$ in $H^{\prime}(\mathbf{A}), g$ in $\left.\widetilde{H}(\mathbf{A})\right)$. Define a functional $L$ on the space of $\tilde{\pi}$ by

$$
L(f)=\sum_{u \in F^{\times} / F^{\times 2}} l\left(f\left(\begin{array}{ll}
u & 0 \\
0 & 1
\end{array}\right)\right) .
$$

The sum converges since $f$ is compactly supported modulo $H^{\prime}(\mathbf{A})$, and as $\left.L(\tilde{\pi}(g) f)=\sum l\left(f\left(\begin{array}{ll}u & 0 \\ 0 & 1\end{array}\right) g\right)\right)$ and $l$ is $H^{\prime}(F)$-invariant, it follows that $L$ is $\widetilde{H}(F)$-invariant. The map intertwining $V_{\tilde{\pi}}$ and $L(\tilde{H}(F) \backslash \widetilde{H}(\mathbf{A}))$ is defined by $f \rightarrow \phi_{f}(g)=L(\tilde{\pi}(g) f)$. It is clearly nonzero. The induced representation $\tilde{\pi}$ is reducible, and we deduce that one of its irreducible components is 
automorphic. It is clear that the restriction of a cuspidal $\tilde{\pi}$ to $H(\mathbf{A})$ is also cuspidal.

\section{MAIN THEOREMS}

Let $F$ be a global field. Fix a place $u$ to be nonarchimedean, unless otherwise specified. Put $H=\mathrm{SL}(2), H_{1}=\operatorname{PGL}(2), G=\operatorname{PGL}(3)$. An irreducible $\sigma$-invariant $G_{u}$-module $\pi_{u}$ is called $\sigma$-elliptic if its twisted character is not identically zero on the $\sigma$-elliptic regular set.

2.1. Proposition. Given a supercuspidal $\pi_{0 u}^{\prime}$ there exists (i) a $\sigma$-invariant $\sigma$ stable $\sigma$-elliptic nondegenerate tempered $\pi_{u}$, which is not Steinberg, and (ii) for each $\pi_{0 u}$ a nonnegative integer $n\left(\pi_{0 u}\right)$, with $n\left(\pi_{0 u}^{\prime}\right) \neq 0$, which depends only on the packet of $\pi_{0 u}$, and which is equal to 0 if $\pi_{0 u}$ is one-dimensional or special, such that for all matching $f_{u}, f_{0 u}$ we have

$$
\operatorname{tr} \pi_{u}\left(f_{u} \times \sigma\right)=\sum n\left(\pi_{0 u}\right) \operatorname{tr}\left\{\pi_{0 u}\right\}\left(f_{0 u}\right) .
$$

The sum is absolutely convergent. Given an open compact subgroup $C_{u}$ of $H_{u}=$ $H\left(F_{u}\right)$, there are only finitely many terms $\pi_{0 u}$ in the sum which have nonzero $C_{u}$-fixed vector.

Remark. (2.1.1) holds of course when $\pi_{0 u}^{\prime}$ is special. Then $\pi_{u}$ is Steinberg, and the sum consists of $\pi_{0 u}^{\prime}$ alone.

Proof. We may assume that $F$ is totally imaginary. Let $\pi_{0}^{\prime}$ be a cuspidal representation of $H(\mathbf{A})$ which has the component $\pi_{0 u}^{\prime}$ at $u$, its component at another finite place $w$ is special, and it is unramified at any other finite place. It is easy to construct such $\pi_{0}^{\prime}$ using the trace formula for $H$, and a function $f_{0}=\otimes f_{0 v}$ whose component at $u$ is a matrix coefficient of $\pi_{0 u}$, at $w$ it is a pseudo-coefficient of the special representation, at the other finite places it is the unit element of the Hecke algebra, and at the infinite places the component has small compact support near the identity.

Apply Proposition 1.7 with $\pi_{0}^{\prime}$ and the set $V=\{u, w\}$. Take $f_{0 w}$ to be a pseudo-coefficient of the special representation. Hence all terms on the right of (1.7.1) belong to $I_{0}$. We obtain the right side of (2.1.1). If we take $f_{0 u}$ to be a matrix-coefficient of $\pi_{0 u}^{\prime}$ we obtain a positive integer (the multiplicity of $\pi_{0}^{\prime}$ in the cuspidal spectrum of $\left.H(\mathbf{A})\right)$ on the right of (1.7.1). Hence there exists a (necessarily unique under the conditions of (1.7)) term $\pi$ on the left of (1.7.1). If $f_{w}$ is a function which satisfies (1.6.1) and matches a pseudocoefficierit of the special representation, then $\left\langle\chi_{\pi_{w}}, \chi_{\mathbf{S t}_{w}}\right\rangle_{e}=\operatorname{tr} \pi_{w}\left(f_{w} \times \sigma\right) \neq 0$ by the orthogonality relations [II, (3.4)], and the component of $\pi$ at $w$ is Steinberg. Hence $\pi$ is a $\sigma$-invariant cuspidal representation in $I$ of (1.7.1), and (2.1.1) follows. Note that $\pi_{u}$ is $\sigma$-stable since the right side depends only on $f_{0 u}$. Moreover, $\pi_{u}$ is nondegenerate since $\pi$ is cuspidal. Consequently $\pi_{u}$ is tempered, since it is $\sigma$-elliptic and nondegenerate.

Further, $\pi_{u}$ is not Steinberg. Indeed, if it is, then it is the lift of the special $\pi_{0 u}^{\prime \prime}$, and (2.1.1) becomes $\operatorname{tr} \pi_{0 u}^{\prime \prime}\left(f_{0 u}\right)=\sum n\left(\pi_{0 u}\right) \operatorname{tr}\left\{\pi_{0 u}\right\}\left(f_{0 u}\right)$. Taking $f_{0 u}$ to be a matrix-coefficient of $\pi_{0 u}^{\prime}$ we conclude that $n\left(\pi_{0 u}^{\prime}\right)$ is 0 . Moreover, no $\pi_{0 u}$ is special. Indeed, taking $f_{0 u}$ to be a pseudo-coefficient of $\pi_{0 u}$, we obtain $n\left(\pi_{0 u}\right)$ on the right of (2.1.1), and on the left 0 , by the twisted orthogonality relations of [II, Lemma 3.6.1]. 
The final claim follows from Harish-Chandra's theorem quoted in (1.7.6).

Let $K_{u}$ be a quadratic field extension of $F_{u}$; denote by $K_{u}^{1}$ the group of elements in $K_{u}$ whose norm in $F_{u}$ is one (as in (1.2.2)).

2.2. Proposition. Given a character $\theta_{u}$ of $C_{K_{u}}^{1}=K_{u}^{1}$ there are nonnegative integers $n\left(\pi_{0 u}\right)$ with $n\left(\pi_{0}\left(\theta_{u}\right)\right)=1$, and a complex number $c \neq 0$, with

$$
c \operatorname{tr} \pi_{u}^{\prime}\left(f_{u} \times \sigma\right)=\sum n\left(\pi_{0 u}\right) \operatorname{tr}\left\{\pi_{0 u}\right\}\left(f_{0 u}\right),
$$

for matching $f_{0 u}, f_{u}$, where $\pi_{u}^{\prime}=I\left(\pi^{*}\left(\theta_{u}\right), \chi_{u}\right)$ in the notations of (1.2.2). The sum is absolutely convergent and includes neither the trivial nor the special representation.

Remark. Here $u$ may be a real place.

Proof. If $u$ is nonarchimedean we work with a totally imaginary $F$. If $u$ is real take $F=\mathbf{Q}$ and imaginary quadratic $K$. The claim is clear if (1) $\theta_{u}=1$, since then $\pi_{0}\left(\theta_{u}\right)$ is an induced representation, $c=1$ and there is only one term in the sum, or (2) $u$ splits in $K / F$. If $\theta_{u} \neq 1$ we fix a finite inert place $w \neq u$ and a character $\theta_{w}$ of $K_{w}^{1}$ with $\theta_{w}^{2} \neq 1$. Let $\theta$ be a character of $C_{K}^{1}$ which has the above components at $u$ and $w$, and all its components at the finite $v \neq u, w$ are unramified, except perhaps at a place $v^{\prime} \neq u, w$ which splits in $K$ if $u$ is real. It is easy to construct such $\theta$ using the trace (or Poisson summation) formula for the pair $C_{K}^{1}$ and $K^{1}$, and a function $f=\otimes f_{v}$ with $f(1) \neq 0$; with $f_{u}=\bar{\theta}_{u} ; f_{w}=\bar{\theta}_{w} ; f_{v}$ is the characteristic function of the maximal compact subgroup of $K_{v}^{1}$ for all finite $v \neq u, w, v^{\prime}$; and $f_{v}$ supported on a small compact neighborhood of 1 if $v$ is complex (when $u$ is finite) or $v$ is $v^{\prime}$ (if $u$ is real).

Since $\theta_{w}^{2} \neq 1$ we have $\theta^{2} \neq 1$. We apply Proposition 1.7 with $\pi=$ $I(\pi(\theta / \bar{\theta}), \chi)$ in $I^{\prime}$ on the left. Then $\left\{\pi_{0}(\theta)\right\}$ appears on the right, in $I_{0}^{\prime}$. To obtain (2.2.1) we apply the argument of (1.7.5) at all split places (including $v^{\prime}$ or the complex places), and take $f_{0 w}$ to be a matrix coefficient of a member of the packet $\left\{\pi_{0}\left(\theta_{w}\right)\right\}$. This explains the appearance of $c \neq 0$. We may choose $f_{w}$ to satisfy (1.6.1).

2.3. Proposition. Only square-integrable $\pi_{0}$ appear in the sum of (2.1.1). Moreover, the sum is finite. The same conclusion holds for (2.2.1) if $u$ is real and $\theta_{u} \neq 1$.

Proof. Recall that since the left side of (2.1.1) depends only on the stable orbital integrals of $f_{u}$, namely on $f_{0 u}$, it is clear that the character $\chi_{u}$ of $\pi_{u}$ is a $\sigma$ stable function, namely it depends only on the stable $\sigma$-conjugacy classes. We now use the (immediate) twisted analogue of [K, Theorems $C$ and $D]$. Then there exists a function $f_{u}^{\prime}$ whose orbital integrals vanish on the $\sigma$-split regular set, and ' $\Phi_{f_{u}^{\prime}}(\delta)=\chi_{u}(\delta)$ on the $\sigma$-elliptic regular set. We write ' $\Phi_{f_{u}}$ for the function on the $\sigma$-elliptic regular set whose value at $\delta$ is $|T| \Phi_{f_{u}}(\delta)$, where $|T|$ is the volume of the centralizer of $N \delta$ in $H\left(F_{u}\right)$. By [I, $\left.\S 3\right]$, there is a function $f_{0 u}^{\prime}$ matching $f_{u}^{\prime}$. We can choose $f_{0 u}^{\prime}$ to have vanishing unstable orbital integrals. Moreover, there exist finitely many tempered elliptic $\pi_{0 u}$, and nonzero complex numbers $c\left(\pi_{0 u}\right)$, so that ${ }^{\prime} \Phi_{f_{0 u}^{\prime}}(\gamma)=\sum c\left(\pi_{0 u}\right) \chi_{\pi_{0 u}}(\gamma)$ on the regular elliptic set. Consequently

$$
\sum c\left(\pi_{0 u}\right) \operatorname{tr} \pi_{0 u}\left(f_{0 u}\right)=\sum n\left(\pi_{0 u}\right) \operatorname{tr}\left\{\pi_{0 u}\right\}\left(f_{0 u}\right),
$$


for all $f_{0 u}$ whose orbital integrals vanish on the regular split set. Evaluating both sides at a pseudocoefficient of any square-integrable representation we conclude that there are only finitely many elliptic (square-integrable or trivial) representations on the right of (2.1.1).

It remains to show that only square-integrable $\pi_{0 u}$ appear in (2.1.1). In the nonarchimedean case the $\left\{\pi_{0 u}\right\}$ on the right are packets which consist either of supercuspidal $\mathrm{H}_{0 u}$-modules, or of the irreducible constituents in the composition series of an induced $H_{0 u}$-module. Fix a character $\mu$ of $A_{0}\left(R_{u}\right) \simeq R_{u}^{\times}$, and let $f_{0 u}$ be an $(n, \mu)$-regular function with $n \geq 1$. Then $\operatorname{tr}\left\{\pi_{0 u}\right\}\left(f_{0 u}+\bar{f}_{0 u}\right)$ vanishes unless $\left\{\pi_{0 u}\right\}$ is the set of constituents of $I_{0}\left(\mu^{\prime}\right)$ with $\mu^{\prime}=\mu$ on $R_{u}^{\times}$, where its value is $z^{n}+z^{-n}$ (times two if $\mu^{2}=1$ ), where $z=\mu^{\prime}(\boldsymbol{\pi})$. Hence the right side takes the form $\sum_{i} c_{i}\left(z_{i}^{n}+z_{i}^{-n}\right)$. The sum is absolutely convergent, and $\left|z_{i}\right|=1$, or $z_{i}=\bar{z}_{i}$, and $q_{u}^{-1}<\left|z_{i}\right|<q_{u}$ (by unitarity). It is also clear from the last assertion of Proposition 2.1 that this sum is finite. On the left, since $\pi_{u}$ is $\sigma$-elliptic and nondegenerate, if the value of $\operatorname{tr} \pi_{u}\left(f_{u} \times \sigma\right)$ is not zero then $\pi_{u}$ is induced from the special representation of a maximal parabolic subgroup of $G_{u}$, and $\operatorname{tr} \pi_{u}\left(f_{u} \times \sigma\right)$ is equal to $q_{u}^{-n / 2}$. Applying the Stone-Weierstrass theorem as in (1.6.3) we conclude that $c_{i}=0$ for all $i$. In particular each packet $\left\{\pi_{0 u}\right\}$ on the right consists of supercuspidals, the sum is finite, and $\pi_{u}$ on the left is not induced from the special representation of the maximal parabolic. When $F_{u}$ is real, the sum is again absolutely convergent. The packet $\left\{\pi_{0 u}\right\}$ either consists of square-integrables, and then $\operatorname{tr}\left\{\pi_{0 u}\right\}\left(f_{0 u}\right)=z^{n}$ for suitable $f_{0 u}=f_{0 u}(n)$ and $z=z\left(\left\{\pi_{0 u}\right\}\right)$ with $|z|<1$, or $\operatorname{tr}\left\{\pi_{0 u}\right\}\left(f_{0 u}\right)=\operatorname{tr}\left[I_{0}(\mu)\right]\left(f_{0 u}\right)$ takes the form $z^{n}+z^{-n}$. This case can be left to the reader (cf. [L, pp. 218221]).

Remark. The above arguments imply also that the sum of (2.2.1) is finite and consists of square-integrable $\pi_{0 u}$ when $\theta_{u} \neq 1$.

2.3.1. Corollary. If $\left\{\pi_{0 u}\right\} \neq\left\{\pi_{0}\left(\theta_{u}\right)\right\}$ appears on the right of $(2.2 .1)$ then it is extraordinary (namely it is supercuspidal but not of the form $\pi_{0}\left(\theta_{u}^{\prime}\right)$ for any character $\theta_{u}^{\prime}$ of $K_{u}^{\prime}$ ). In particular, if $F_{u}$ is real, or has odd residual characteristic, then $\left\{\pi_{0}\left(\theta_{u}\right)\right\}$ is the only term in the sum of (2.2.1).

Proof. This follows from the finiteness of the sum, and the twisted orthogonality relations for characters. Indeed, if $\left\{\pi_{0 u}\right\} \neq\left\{\pi_{0}\left(\theta_{u}\right)\right\}$ appears on the right of (2.2.1), namely $n\left(\pi_{0 u}\right) \neq 0$, and $\left\{\pi_{0 u}\right\}=\left\{\pi_{0}\left(\theta_{u}^{\prime}\right)\right\}, \theta_{u}^{\prime} \neq \theta_{u}$, then (2.2.1) for $\theta_{u}^{\prime}$ implies that $c^{\prime}\left(\chi\left(\pi_{u}^{\prime \prime}\right)\right)(\delta)=\sum n^{\prime}\left(\pi_{0 u}\right) \chi\left(\left\{\pi_{0 u}\right\}\right)(N \delta)$ with $\pi_{u}^{\prime \prime}=I\left(\pi^{*}\left(\theta_{u}^{\prime}\right), \chi_{u}^{\prime}\right)$ and $n^{\prime}\left(\pi_{0}\left(\theta_{u}^{\prime}\right)\right) \neq 0$. Since $\chi\left(\pi_{u}^{\prime}\right)$ is orthogonal to $\chi\left(\pi_{u}^{\prime \prime}\right)$, we obtain

$$
0=\sum n\left(\pi_{0 u}\right) n^{\prime}\left(\pi_{0 u}\right) \geq n\left(\pi_{0}\left(\theta_{u}^{\prime}\right)\right) n^{\prime}\left(\pi_{0}\left(\theta_{u}^{\prime}\right)\right)>0 .
$$

This contradiction proves the first claim. In the real case and in the case of odd residual characteristic, all square-integrable representations are special or of the form $\pi_{0}\left(\theta_{u}\right)$.

2.3.2. Corollary. Given a square-integrable $\pi_{0 u}^{\prime}$ as in (2.1), the relation (2.1.1) determines the tempered $\pi_{u}$ uniquely.

Proof. Suppose we also have $c \operatorname{tr} \pi_{u}^{\prime}\left(f_{u} \times \sigma\right)=\sum m\left(\pi_{0 u}\right) \operatorname{tr}\left\{\pi_{0 u}\right\}\left(f_{0 u}\right)$ for all matching $\left(f_{u}, f_{0 u}\right)$, where $c \neq 0$ and $m\left(\pi_{0 u}\right)$ are nonnegative integers with $m\left(\pi_{0 u}^{\prime}\right) \neq 0$, then the orthogonality relations on the right [II, (2.1.1)] and the 
twisted orthogonality relations on the left [II, (3.6.1)], imply that $\pi_{u}^{\prime}$ is equivalent to $\pi_{u}$.

2.3.3. Corollary. If $\pi_{u}$ on the left of (2.1.1) is supercuspidal then $\pi_{0 u}^{\prime}$ is extraordinary, it is the only term on the right of (2.1.1), and $n\left(\pi_{0 u}^{\prime}\right)=1$. The residual characteristic of $u$ is even.

Proof. For a supercuspidal $\pi_{u}$ we have twisted orthonormality relations for its character [II, (3.3.1)], namely $\left\langle\chi_{\pi_{u}}, \chi_{\pi_{u}}\right\rangle_{e}=1$ in the notations of [II, (3.6)]. On the right the orthogonality relations for characters [II, (2.1.1)], imply that $\left\langle\sum n\left(\pi_{0 u}\right) \chi_{\left\{\pi_{0 u}\right\}}, \sum n\left(\pi_{0 u}\right) \chi_{\left\{\pi_{0 u}\right\}}\right\rangle$ is equal to $\sum n\left(\pi_{0 u}\right)^{2}\left[\left\{\pi_{0 u}\right\}\right]$, where $\left[\left\{\pi_{0 u}\right\}\right]$ denotes the number of elements in the packet $\left\{\pi_{0 u}\right\}$. It follows that the sum consists of $\pi_{0 u}^{\prime}$ alone, with coefficient $n\left(\pi_{0 u}^{\prime}\right)=1$, and the packet of $\pi_{0 u}^{\prime}$ consists of a single representation. Since it is square-integrable but not special, it is extraordinary.

2.4. Proposition. Suppose that $f_{u}^{\prime}$ satisfies (1.6.1), namely its unstable orbital integral vanishes on the $\sigma$-regular set. Then $\operatorname{tr} I\left(\pi_{1 u}, 1\right)\left(f_{u}^{\prime} \times \sigma\right)$ is zero for any irreducible representation $\pi_{1 u}$ of $H_{1}\left(F_{u}\right)$.

Remark. Here $u$ is allowed to be archimedean.

Proof. This is obvious if $\pi_{1 u}$ is an induced representation, since then the (twisted) character of $I\left(\pi_{1 u}, 1\right)$ is supported on the $\sigma$-split set, where the $\sigma$-orbital integral of $f_{u}^{\prime}$ is assumed to be zero. The case of one-dimensional representations follows from that of special ones, hence we assume that $\pi_{1 u}$ is square-integrable. Let $\pi_{1}$ be a cuspidal representation of $H_{1}(\mathbf{A})$ whose component at $u$ is the above, and whose component at a fixed finite place $w \neq u$ of odd residual characteristic is supercuspidal. We may choose $F$ so that all its archimedean places, except possibly $u$, are complex. Apply Proposition 1.7 so that $I\left(\pi_{1}, 1\right)$ is the only term on the left, in $I_{1}^{\prime}$, with $u_{0}=u$. Assuming that $\operatorname{tr} I\left(\pi_{1 u}, 1\right)\left(f_{u}^{\prime} \times \sigma\right) \neq 0$ we conclude that the character of $I\left(\pi_{1 u}, 1\right)$ is $\sigma$-stable and $\sigma$-elliptic, and there is a term $\pi_{0}$ on the right of (1.7.1) whose component at $u$ is elliptic. We take $f_{0 u}$ to be a pseudo-coefficient of $\pi_{0 u}$; for each finite $v \neq u, w$ we take $f_{0 v}$ to be the characteristic function of a small compact open subgroup of $H\left(F_{v}\right)$, as in Lemma 1.9. We obtain the identity

$$
c \operatorname{tr} I\left(\pi_{1 w}, 1\right)\left(f_{w} \times \sigma\right)=\sum n\left(\pi_{0 w}\right) \operatorname{tr}\left\{\pi_{0 w}\right\}\left(f_{0 w}\right),
$$

with the properties of (2.2.1). The sum is finite and made of supercuspidals by the arguments of (2.3). But $w$ has an odd residual characteristic, hence any supercuspidal packet $\left\{\pi_{0 w}\right\}$ is of the form $\left\{\pi_{0}\left(\theta_{w}\right)\right\}$, which defines the representation $I\left(\pi\left(\theta_{w} / \bar{\theta}_{w}\right), \chi_{w}\right)$ by (2.3.1), and we obtain a contradiction to Corollary 2.3.1 on using the orthogonality relations for characters (on the right side) and twisted characters [II, (2.6.1)] on the left side.

2.5. Proposition. Suppose $\pi_{u}$ is a $\sigma$-invariant supercuspidal representation which is not $\sigma$-unstable. Then it is the lift of an extraordinary $\pi_{0 u}$, hence $\sigma$ stable.

Proof. Recall that a $\sigma$-conjugacy class function $\chi$ is called $\sigma$-unstable if $\chi(\tilde{\delta})=$ $-\chi(\delta)$ for any $\sigma$-ellipitc regular stably $\sigma$-conjugate but non- $\sigma$-conjugate $\delta$ and $\tilde{\delta}$ in $G\left(F_{u}\right)$. We use the Deligne-Kazhdan trace formula with a function $f=$ $\otimes f_{v}$ whose component at $u$ is a matrix coefficient of $\pi_{u}$, and its component 
at some finite $u_{0} \neq u$ satisfies (1.6.1) and is supported on the $\sigma$-ellipitc regular set, to prove the existence of a $\sigma$-invariant cuspidal $\pi$ whose component at $u$ is ours, and whose component at $u_{0}$ is $\sigma$-elliptic and $\sigma$-stable. Here we use the assumption that $\pi_{u}$ is not $\sigma$-unstable to deduce the existence of a $\sigma$ elliptic regular $\delta$ with $\Phi_{f_{u}}^{\text {st }}(\delta) \neq 0$. This $\pi$ appears in $I$ of $(1.7 .1)$, and we use Proposition 1.7 with $f_{0 v}$ as in (1.9) at each $v \neq u, u_{0}$ of $V$ in (1.7), and a pseudo-coefficient $f_{0 u_{0}}$ of a square-integrable component at $u_{0}$ of a $\pi_{0}$ which must appear on the right of (1.7.1). We obtain (2.1.1) where the left side is perhaps replaced by $c \operatorname{tr} \pi_{u}\left(f_{u} \times \sigma\right)$, where $c \neq 0$. The sum on the right is finite and consists of supercuspidal $\pi_{0 u}$, by (2.3). We take one of the $\pi_{0 u}$ which appears on the right, and obtain (2.1.1) for it. By (2.3.2) we obtain again $\pi_{u}$ on the left of (2.1.1), now with coefficients $c=1 ;(2.3 .3)$ implies that $\pi_{0 u}$ is the only term on the right of (2.1.1), and it extraordinary with coefficient 1 .

2.6. Proposition. Only one packet, namely $\left\{\pi_{0 u}^{\prime}\right\}$, appears on the right of (2.1.1).

Proof. Suppose that in addition to $\left\{\pi_{0 u}^{\prime}\right\}$ there appears also the (necessarily supercuspidal) packet $\left\{\pi_{0 u}^{\prime \prime}\right\}$ on the right of $(2.1 .1)$, with $n\left(\pi_{0 u}^{\prime \prime}\right) \neq 0$. Denote by $f\left(\pi_{0 u}\right)$ a matrix coefficient of a supercuspidal $\pi_{0 u}$, and by $f\left(\left\{\pi_{0 u}\right\}\right)$ the sum of $f\left(\pi_{0 u}\right)$ over the $\pi_{0 u}$ in the packet $\left\{\pi_{0 u}\right\}$. Write $f_{0 u}$ for $n\left(\pi_{0 u}^{\prime \prime}\right) f\left(\left\{\pi_{0 u}^{\prime}\right\}\right)-$ $n\left(\pi_{0 u}^{\prime}\right) f\left(\left\{\pi_{0 u}^{\prime \prime}\right\}\right)$. Then the function $f_{0 u}$ is not uniquely determined, but its orbital integrals are. Moreover, its orbital integrals are stable conjugacy class functions, since ${ }^{\prime} \Phi\left(f\left(\left\{\pi_{0 u}\right\}\right)\right)=\chi_{\left\{\pi_{0 u}\right\}}$ on the elliptic regular set, and these orbital integrals are zero on the split regular set. Denote by $f_{u}$ a function on $G_{u}$ matching $f_{0 u}$. We can choose $f_{u}$ to have zero unstable orbital integrals by [I, §3], since its (twisted) orbital integrals are zero on the $\sigma$-regular split set. It is clear from the orthogonality relations for characters that we have $\operatorname{tr} \pi_{u}\left(f_{u} \times \sigma\right)=0$ for every $\sigma$-invariant $G_{u}$-module $\pi_{u}$. Indeed, if $\operatorname{tr} \pi_{u}\left(f_{u} \times \sigma\right) \neq 0$ then $\pi_{u}$ can be only the representation of $G_{u}$ determined by $\left\{\pi_{0 u}^{\prime}\right\}$ and $\left\{\pi_{0 u}^{\prime \prime}\right\}$, but the choice of $f_{0 u}$ guarantees that $\operatorname{tr} \pi_{u}\left(f_{u} \times \sigma\right)=0$ for this $\pi_{u}$. Then Proposition 1.8 shows that $f_{u}$ has vanishing (twisted stable) orbital integrals, hence $f_{0 u}$ has vanishing orbital integrals, and we conclude that $n\left(\pi_{0 u}^{\prime \prime}\right) \chi_{\left\{\pi_{0 u}^{\prime}\right\}}=n\left(\pi_{0 u}^{\prime}\right) \chi_{\left\{\pi_{0 u}^{\prime \prime}\right\}}$ on the elliptic regular set. This contradicts the orthogonality relations for characters, and the proposition follows.

Remark. Analogous discussion can be carried out in the real case on using pseudo-coefficients instead of matrix-coefficients.

2.7. Corollary. For each character $\theta_{u}$ of $K_{u}^{1}$ there is a positive integer $n_{u}=$ $n_{u}\left(\theta_{u}\right)$ so that $\operatorname{tr} \pi_{u}\left(f_{u} \times \sigma\right)=n_{u} \operatorname{tr}\left\{\pi_{0}\left(\theta_{u}\right)\right\}\left(f_{0 u}\right)$ for all matching $f_{u}, f_{0 u}$, where $\pi_{u}=I\left(\pi^{*}\left(\theta_{u}\right), \chi_{u}\right)$, and $\chi_{u}$ is the character of $F_{u}^{\times}$with kernel $N K_{u}^{\times}$.

Proof. This follows from (2.2), (2.3.2), and (2.6).

2.7.1. Corollary. Suppose that $\pi_{0 u}$ is an extraordinary (not of the form $\pi\left(\theta_{u}\right)$ ) representation of $H\left(F_{u}\right)$. Then it lifts to a supercuspidal representation $\pi_{u}$ of $G\left(F_{u}\right)$.

Remark. (1) Extraordinary representations exist only in even residual characteristic. (2) $\pi_{u}$ is $\sigma$-invariant, $\sigma$-stable and $\sigma$-elliptic.

Proof. This follows from (2.3.2), (2.7), (2.4) and (2.5). 
2.8. Theorem. Let $F$ be a local field. (1) The one-dimensional, special, extraordinary, type $\pi_{0}(\theta)$, representation of $H(F)$, lift (respectively) to the onedimensional, Steinberg, supercuspidal, $I(\pi(\theta / \bar{\theta}), \chi)$, representations of $G(F)$; local lifting is defined in [II, (2.3)]. (2) $A$ o-invariant admissible irreducible representation $\pi$ of $G(F)$ is a lift of a packet $\left\{\pi_{0}\right\}$ of $H(F)$ precisely when it is $\sigma$-stable. Thus $\pi$ is a lift unless it is of the form $I\left(\pi_{1}, 1\right)$, where $\pi_{1}$ is an elliptic representation of $H_{1}(F)$, or it is $\sigma$-unstable and supercuspidal. ${ }^{1}$

Proof. This follows from [II, (2.9)] (case of special representations), (2.7.1) and (2.5) (extraordinary case), (2.4) (case of $I\left(\pi_{1}, 1\right)$ ), as well as (1.4) (list of $\sigma$ invariant representations), once we show that $\pi_{0}\left(\theta_{u}\right)$ lifts to $I\left(\pi\left(\theta_{u} / \bar{\theta}_{u}\right), \chi_{u}\right)$, namely that the $n_{u}$ of (2.7) is equal to 1 . For that we use a totally imaginary global field, take a global character $\theta$ whose component at $u$ is our local character, and apply $(1.7)$ where $I(\pi(\theta / \bar{\theta}), \chi)$ is the only (global) term on the left. The finite set $V$ of (1.7) can be assumed to have no split (in $K / F$ ) places by the argument of Lemma 1.7.5, and $\theta_{v} \neq 1$ on $K_{v}^{1}$ for all $v$ in $V$ (including our $u$ ). Using (2.7) the identity (1.7.1) of (1.7) reads

$$
\prod n_{v} \operatorname{tr}\left\{\pi_{0}\left(\theta_{v}\right)\right\}\left(f_{0 v}\right)=\prod \operatorname{tr}\left\{\pi_{0}\left(\theta_{v}\right)\right\}\left(f_{0 v}\right)+\sum n\left(\pi_{0}\right) \prod \operatorname{tr}\left\{\pi_{0 v}\right\}\left(f_{0 v}\right) .
$$

The sum is over discrete-series packets $\pi_{0}$ which are inequivalent to our $\pi_{0}(\theta)$, and the coefficient of the first term on the right is one as noted in the discussion of the terms $I_{0}^{\prime}$ and $I_{0}^{\prime \prime}$ in (1.3). It suffices to choose $f_{0 v}$ to be a coefficient of a supercuspidal member of $\left\{\pi_{0}\left(\theta_{v}\right)\right\}$ for each $v$ in $V$ to conclude that the product of the positive integers $n_{v}$ is one, hence $n_{v}$ is 1 for all $v$. Note that the matching $f_{v}$ can be chosen to satisfy (1.6.1). From the conclusion for the $p$-adic places we conclude the claim for real $u$ on using $Q$ for our global field, and an imaginary quadratic extension of it for $K$.

Remark. It can be shown that $\sigma$-invariant supercuspidal representation cannot be $\sigma$-unstable, in fact they must be $\sigma$-stable and lifts of extraordinary $\pi_{0}$, once we have the statement $f_{1}^{0}=\lambda_{1}^{*}\left(f^{0}\right)$ (relating the unit elements $f_{1}^{0}, f^{0}$ in the Hecke algebras $\mathbf{H}_{1}, \mathbf{H}$ of $H_{1}(F)$ and $G(F)$; see [I, §4]). This is proven in [J]. Given this statement, the above methods are extended in [VI] to yield the following global theorem unconditionally. For now we prove the following

2.9. Theorem. Let $F$ be a number field. (1) Any $\sigma$-invariant cuspidal representation $\pi$, with a component which is the lift of a square-integrable $\pi_{0 u}$, is a lift of a unique cuspidal packet $\left\{\pi_{0}\right\}$ of $H(\mathbf{A})$, whose members have a squareintegrable component at $u$. (2) If the discrete-series $\pi$ is a lift $\left(=\lambda\left(\left\{\pi_{0}\right\}\right)\right)$ then it has no component of the form $I\left(\pi_{1 v}, 1\right)$ where $\pi_{1 v}$ is elliptic. (3) Any packet $\left\{\pi_{0}\right\}$ which contains a cuspidal representation with an elliptic component lifts to an automorphic representation $\pi$ of $G(\mathbf{A})$. (4) $\pi$ is cuspidal, unless $\pi_{0}$ is one-dimensional (and then $\pi$ is one-dimensional), or $\pi_{0}$ is of the form $\pi_{0}(\theta)$ associated with $\operatorname{Ind}\left(\theta ; W_{K}, W_{F}\right)$, where $\pi$ is $I(\pi(\theta / \bar{\theta}), \chi)$. (5) Each cuspidal $\pi_{0}$ with an elliptic component occurs only once in the cuspidal spectrum of $L(H(F) \backslash H(\mathbf{A}))$. (6) If $\pi_{0}^{\prime}$ is cuspidal with an elliptic component at a place where $\pi_{0}$ has an elliptic component, and $\pi_{0 v}^{\prime} \simeq \pi_{0 v}$ for almost all $v\left(\pi_{0}\right.$ as in (5)), then $\left\{\pi_{0}\right\}=\left\{\pi_{0}^{\prime}\right\}$.

\footnotetext{
${ }^{1}$ It will be interesting to give a direct local proof (not using the trace formulae) that every $\sigma$-invariant supercuspidal $G(F)$-module $\pi$ is $\sigma$-stable.
} 
Remark. (5) is multiplicity one theorem for (cuspidal representations with an elliptic component of) $H=\mathrm{SL}(2)$. (6) is a rigidity theorem for such packets of $\operatorname{SL}(2, \mathbf{A})$.

Proof. To prove (1) we form (1.7.1) under the conditions of (1.7), where $\pi$ is the only contribution on the left. Each component of $\pi$ is clearly $\sigma$-stable, hence a lift of some $\pi_{0 v}$, and the argument of (1.7.5) implies that the component at $v \neq u$ of any $\pi_{0}$ which appears on the right side of (1.7.1) and (1.7) lifts to $\pi_{v}$. At $u$ we use $f_{u}$ which satisfies (1.6.1) and matches a pseudocoefficient $f_{0 u}$ of a square-integrable $\pi_{0 u}$ which lifts to $\pi_{u}\left(\pi_{0 u}\right.$ exists by our assumption). (1) follows. (2) is clear from (2.4). (3) follows on using (1.7) and (1.9), and (4), (5), (6) are similarly immediate.

2.10. Corollary [GJ]. If a unitary cuspidal representation $\tilde{\pi}_{0}$ of $\mathrm{GL}(2, \mathbf{A})$ with a square-integrable component has a local component $\tilde{\pi}_{0 v}$ of the form $I_{0 v}\left(\nu_{v}^{t}, \nu_{v}^{-t}\right), t \geq 0$, then $t<\frac{1}{4}$.

Proof. The restriction $\left\{\pi_{0}\right\}$ of $\tilde{\pi}_{0}$ to $H(\mathbf{A})$ is a discrete-series packet with a square-integrable component, which lifts to an automorphic representation $\pi$ of $G(\mathbf{A})$. In particular $\tilde{\pi}_{0}\left(\nu_{v}^{t}, \nu_{v}^{-t}\right)$ lifts to $I\left(\nu_{v}^{2 t}, 1, \nu_{v}^{2 t}\right)$, which is unitary only if $2 t<\frac{1}{2}$. A strict inequality holds since our cuspidal $\pi$ cannot have a component at $v$ which lies in the composition series of $I\left(\nu_{v}^{1 / 2}, 1, \nu_{v}^{-1 / 2}\right)$, by Proposition 2.4.

2.10.1. For any representation $\tilde{\pi}_{0 v}$ of $\mathrm{GL}\left(2, F_{v}\right)$ and character $\chi_{v}$ of $F_{v}^{\times}$put

$$
L_{2}\left(s, \tilde{\pi}_{0 v}, \chi_{v}\right)=L\left(s, \tilde{\pi}_{0 v} \otimes \chi_{v} \times \check{\tilde{\pi}}_{0 v}\right) / L\left(s, \chi_{v}\right),
$$

and

$$
\varepsilon_{2}\left(s, \tilde{\pi}_{0 v}, \chi_{v} ; \psi_{v}\right)=\varepsilon\left(s, \tilde{\pi}_{0 v} \otimes \chi_{v} \times \check{\tilde{\pi}}_{0 v} ; \psi_{v}\right) / \varepsilon\left(s, \chi_{v} ; \psi_{v}\right) .
$$

Here $\check{\tilde{\pi}}_{0 v}$ is the contragredient of $\tilde{\pi}_{0 v}$ and $\psi_{v}$ is a nontrivial additive character of $F_{v}$. The $L$-functions depend only on the packet $\left\{\pi_{0 v}\right\}$ defined by $\pi_{0 v}$. As in [GJ], we say that $\tilde{\pi}_{0 v}$ L-lifts to a representation $\pi_{v}$ of $G\left(F_{v}\right)$ if $\pi_{v}$ is $\sigma$-invariant and

$$
L\left(s, \pi_{v} \otimes \chi_{v}\right)=L_{2}\left(s, \tilde{\pi}_{0 v}, \chi_{v}\right), \quad \varepsilon\left(s, \pi_{v}, \otimes \chi_{v} ; \psi_{v}\right)=\varepsilon_{2}\left(s, \tilde{\pi}_{0 v}, \chi_{v} ; \psi_{v}\right),
$$

for any character $\chi_{v}$ of $F_{v}^{\times}$. Here $\pi_{v}$ is viewed as a representation of GL $\left(3, F_{v}\right)$ with a trivial central character. Gelbart and Jacquet [GJ, Propositions 3.2, 3.3], showed for nonextraordinary $\tilde{\pi}_{0 v}$ that $\left\{\pi_{0 v}\right\} L$-lifts to the lift $\pi_{v}$ of $\left\{\pi_{0 v}\right\}$. If $\tilde{\pi}_{0}$ is an automorphic representation of $\mathrm{GL}(2, \mathbf{A})$ and $\chi$ is a character of $F^{\times} \backslash \mathbf{A}^{\times}$, then the function $L_{2}\left(s, \tilde{\pi}_{0}, \chi\right)$ is defined to be the product over all $v$ of the $L_{2}\left(s, \tilde{\pi}_{0 v}, \chi_{v}\right)$.

2.10.2. Corollary [GJ]. If $\pi_{0}$ is cuspidal, not of the form $\pi_{0}(\theta)$, and has a square-integrable component, then

$$
L_{2}\left(s, \pi_{0}, \chi\right)=L(s, \pi \otimes \chi)
$$

for any character $\chi$ of $F^{\times} \backslash \mathbf{A}^{\times}$, where $\pi$ is the lift of $\pi_{0}$. Hence $L_{2}\left(s, \pi_{0}, \chi\right)$ is entire for any $\chi$.

Proof. The local factors of the two global products are equal unless $\pi_{v}$ is supercuspidal, but then both local factors are equal to 1 . 
It is easy to deduce from this [GJ, p. 535] that $\pi_{0 v} L$-lifts to its lift $\pi_{v}$ in the remaining case, where $\pi_{v}$ is supercuspidal.

Corollary 2.10 .2 was proved directly using the Rankin-Shimura method in [GJ], where it was used as the key tool to prove that each $\pi_{0 v}$ and $\pi_{0} L$-lift to their lifts, with no conditions on local components. The advantage of the trace formula is in characterizing the image of the lifting, establishing character relations and proving the multiplicity one theorem and the rigidity theorem for $\mathrm{SL}(2)$, in addition to proving the existence of the lifting.

\section{REFERENCES}

[BZ] J. Bernstein and A. Zelevinsky, Induced representations of reductive p-adic groups, Ann. Sci. Ecole Norm Sup. 10 (1977), 441-472.

[B] A. Borel, Automorphic L-functions, Proc. Sympos. Pure Math. vol. 33, part II, Amer. Math. Soc., Providence, R. I., 1979, pp. 27-63. [ $\mathrm{B}^{\prime}$ ] Admissible representations with vectors fixed under an Iwahori subgroup, Invent. Math. 35 (1976), 233-259.

[BJ] A. Borel and H. Jacquet, Automorphic forms and automorphic representations, Proc. Sympos. Pure Math., vol. 33, part I, Amer. Math. Soc., Providence, R. I., 1979, pp. 189-208.

[C] W. Casselman, Characters and Jacquet modules, Math. Ann. 230 (1977), 101-105; see also: P. Deligne, Le support du caractère d'une represéntation supercuspidale, C. R. Acad. Sci. Paris 283 (1976), 155-157.

[Cl] L. Clozel, Characters of non-connected, reductive p-adic groups, Canad. J. Math. 39 (1987), 149-167.

[CL] L. Clozel, J. P. Labesse, and R. Langlands, Seminar at IAS, Princeton, N. J., 1984.

[F] Y. Flicker, $\left[\mathrm{F}_{0}\right]$ : The trace formula and base change for $\mathrm{GL}(3)$, Lecture Notes in Math., vol. 927, Springer, 1982; $\left[\mathrm{F}_{1}\right]$ : The adjoint lifting from $\mathrm{SL}(2)$ to $\mathrm{PGL}(3)$, IHES preprint series, 1981; $\left[\mathrm{F}_{2}\right]$ : Outer automorphisms and instability, Théorie de Nombres, Paris 198081, Progress in Math., vol. 22, Birkhäuser, 1982; $\left[\mathrm{F}_{3}\right]$ : Stable base change for spherical functions, Nagoya Math. J. 106 (1987), 121-142; $\left[\mathrm{F}_{5}\right]$ : Regular trace formula and basechange lifting, Amer. J. Math. 110 (1988), 739-764; $\left[\mathrm{F}_{6}\right]$ : Regular trace formula and base-change for $\mathrm{GL}(n)$, Ann. Inst. Fourier 40 (1990), 1-30.

$\left[\mathrm{F}^{\prime}\right] \quad-,\left[\mathrm{F}_{0}^{\prime}\right]$ : Rigidity for automorphic forms, J. Analyse Math. 49 (1987), 135-202; $\left[\mathrm{F}_{4}\right]$ : Stable and labile base-change for $U(2)$, Duke Math. J. 49 (1982), 691-729; $\left[\mathrm{F}_{1}^{\prime}\right]: L$ packets and liftings for $U(3)$, (Unpublished, Princeton, N. J., 1982); $\left[\mathrm{F}_{2}^{\prime}\right]$ : Unitary quasilifting. preparations, Contemp. Math., vol. 53, Amer. Math. Soc., Providence, R.I., 1986, pp. 119-139; $\left[\mathrm{F}_{3}^{\prime}\right]$ : Unitary quasi-lifting. applications, Trans. Amer. Math. Soc. 294 (1986), 553-565; $\left[\mathrm{F}_{4}^{\prime}\right]$ : Packets and liftings for $U(3)$, J. Analyse Math. $50(1988), 19-63 ;\left[\mathrm{F}_{5}^{\prime}\right]$ : Base change trace identity for $U(3)$, J. Analyse Math. 52 (1989), 39-52.

[I-VI] _ On the symmetric square, [I]: Orbital integrals, Math. Ann. 279 (1987), 173-191; [II]: Definitions and lemmas, Trans. Amer. Math. Soc. 330 (1992), 111-124. [III]: Twisted trace formula, J. Funct. Anal. 98 (1990), 194-210; [IV]: Applications of a trace formula, Trans. Amer. Math. Soc. 330 (1992), 125-152. [V]: (with D. Kazhdan), Unstable local transer, Invent. Math. 91 (1988), 493-504; [VI]: Total global comparison, preprint.

[FK] Y. Flicker and D. Kazhdan, Metaplectic correspondence, Publ. Math. IHES 64 (1987), 53110.

[GJ] S. Gelbart and H. Jacquet, A relation between automorphic representation of $\mathrm{GL}(2)$ and GL(3), Ann. Sci. Ecole Norm. Sup. 11 (1978), 471-541.

[H] Harish-Chandra, Admissible invariant distributions on reductive p-adic groups, Queen's $\mathrm{Pa}-$ pers in Pure and Appl. Math. 48 (1978), 281-346.

[JL] H. Jacquet and R. Langlands, Automorphic form on GL(2), Lecture Notes in Math., vol. 114, Springer, Berlin and New York, 1970.

[JS] H. Jacquet and J. Shalika, On Euler products and the classification of automorphic forms II, Amer. J. Math. 103 (1981), 777-815. 
[J] D. Joyner, On twisted orbital integral identities for PGL(3) over a p-adic field, Canad. J. Math. 42 (1990), 1098-1130.

[K] D. Kazhdan, Cuspidal geometry on p-adic groups, J. Analyse Math. 47 (1986), 1-36; [K']: On lifting, Lie Groups Representations. II, Lecture Notes in Math., vol. 1041, Springer, 1984, pp. 209-249; [K"] : Representations of groups over close local fields, J. Analyse Math. 47 (1986), 175-179.

[LL] J. P. Labesse and R. Langlands, L-indistinguishability for SL(2), Canad, J. Math. 31, (1979), 726-785.

[L] R. Langlands, Base change for GL(2), Ann. of Math. Stud., no. 96, Princeton Univ. Press, 1980; $\left[\mathrm{L}_{1}\right]$ : Les debuts d'une formule des traces stable, Publ. Math. Univ. Paris VII 13 (1980).

[Sh] G. Shimura, On the holomorphy of certain Dirichlet series, Proc. London Math. Soc. 31 (1975), 79-95.

[St] R. Steinberg, Conjugacy classes in algebraic groups, Lecture Notes in Math., vol. 366, Springer, Berlin and New York, 1974.

[Vo] D. Vogan, Gelfand-Kirillov dimension for Harish-Chandra modules, Invent. Math. 48 (1978), 75-98.

[Z] A. Zelevinsky, Induced representations of reductive p-adic groups. II, Ann. Sci. Ecole Norm. Sup. 13 (1980), 165-210.

Department of Mathematics, The Ohio State University, Columbus, Ohio 43210-1174

E-mail address: flicker@function.mps.ohi-state.edu 